\title{
Ventral Midline Thalamus Is Necessary for Hippocampal Place Field Stability and Cell Firing Modulation
}

\author{
Thibault Cholvin, ${ }^{1,2}$ @Vincent Hok, ${ }^{1,2}$ Lisa Giorgi, ${ }^{1,2}$ Franck A. Chaillan, ${ }^{1,2 \star}$ and ${ }^{\circledR B}$ Bruno Poucet ${ }^{1,2 *}$ \\ ${ }^{1}$ Laboratoire de Neurosciences Cognitives and ${ }^{2}$ Federation 3C, CNRS, Aix Marseille University, 13331 Marseille, France
}

The reuniens $(\mathrm{Re})$ and rhomboid $(\mathrm{Rh})$ nuclei of the ventral midline thalamus are reciprocally connected with the hippocampus (Hip) and the medial prefrontal cortex (mPFC). Growing evidence suggests that these nuclei might play a crucial role in cognitive processes requiring Hip-mPFC interactions, including spatial navigation. Here, we tested the effect of ReRh lesions on the firing properties and spatial activity of dorsal hippocampal CA1 place cells as male rats explored a familiar or a novel environment. We found no change in the spatial characteristics of CA1 place cells in the familiar environment following ReRh lesions. Contrariwise, spatial coherence was decreased during the first session in a novel environment. We then investigated field stability of place cells recorded across $5 \mathrm{~d}$ both in the familiar and in a novel environment presented in a predefined sequence. While the remapping capacity of the place cells was not affected by the lesion, our results clearly demonstrated a disruption of the CAl cellular representation of both environments in ReRh rats. More specifically, we found ReRh lesions to produce (1) a pronounced and long-lasting decrease of place field stability and (2) a strong alteration of overdispersion (i.e., firing variability). Thus, in ReRh rats, exploration of a novel environment appears to interfere with the representation of the familiar one, leading to decreased field stability in both environments. The present study shows the involvement of ReRh nuclei in the long-term spatial stability of CA1 place fields.

Key words: hippocampus; lesion; nucleus reuniens; place cells; spatial memory; ventral midline thalamus

Significance Statement

Growing evidence suggest that the ventral midline thalamic nuclei (reuniens and rhomboid) might play a substantial role in various cognitive tasks including spatial memory. In the present article, we show that the lesions of these nuclei impair the spatial representations encoded by CA1 place cells of both familiar and novel environments. First, reduced variability of place cell firing appears to indicate an impairment of attentional processes. Second, impaired stability of place cell representations could explain the long-term memory deficits observed in previous behavioral studies.

\section{Introduction}

Hippocampal place cells are specifically responding to a restricted location in space called the "place field," and each place cell has a different place field in a given environment, such that the place cell population maps the entire space explored by the animal (O'Keefe and Nadel, 1978). This process is thought to be central in spatial navigation, and the hippocampus (Hip) to be the neural

\footnotetext{
Received July 19, 2017; revised 0ct. 12, 2017; accepted Nov. 7, 2017

Author contributions: F.A.C. and B.P. designed research; T.C. and F.A.C. performed research; T.C., V.H., and L.G. analyzed data; T.C., V.H., F.A.C., and B.P. wrote the paper.

Support for this work was provided by the CNRS, Aix-Marseille University, and the Agence Nationale de la Recherche (Grant ANR-14-CE13-0029-02 "THALAME").

*F.A.C. and B.P. contributed equally to this work.

The authors declare no competing financial interests.

Correspondence should be addressed to Dr. Bruno Poucet, Aix Marseille Université, CNRS UMR 7291, Laboratoire

de Neurosciences Cognitives, Fédération 3C, 3, Place Victor Hugo, 13331 Marseille, France. E-mail: bruno.poucet@univ-amu.fr.

DOI:10.1523/JNEUROSCI.2039-17.2017

Copyright $\odot 2018$ the authors $\quad 0270-6474 / 18 / 380158-15 \$ 15.00 / 0$
}

substrate of a cognitive map allowing an allocentric representation of space (Moser et al., 2015). These place cells are pyramidal neurons found in the Cornu Ammonis (CA) areas of the Hip. The nucleus reuniens $(\mathrm{Re})$ of the ventral midline thalamus strongly and specifically projects to CA1, with virtually no projection to the CA3 area (Vertes et al., 2006). Re is also intensely projecting to the medial prefrontal cortex $(\mathrm{mPFC})$ and receives afferents in return from both CA1 and mPFC (Vertes, 2002; McKenna and Vertes, 2004). Together with Re nucleus, the rhomboid (Rh) nucleus form the group of the ventral midline thalamic nuclei. Rh nucleus distributes more widely than the Re nucleus (mainly to the same targets but also to several other regions; Vertes et al., 2006). Using experimental manipulations, such as excitotoxic lesions, reversible inactivations, or optogenetic stimulations, a growing body of data suggests that ReRh might play a substantial role in many cognitive processes, including behavioral flexibility in spatial tasks (Dolleman-van Der Weel et al., 2009; Cholvin et al., 2013), memory specificity and generalization (Xu and Südhof, 2013), spatial working memory (Hembrook and Mair, 2011; 
Hembrook et al., 2012; Layfield et al., 2015; Hallock et al., 2016), goal-directed spatial navigation (Ito et al., 2015), and memory consolidation (Loureiro et al., 2012; Ali et al., 2017).

The role of Re nucleus as a major interface between the mPFC and Hip is further supported by the fact that some of its neurons projecting to CA1 receive direct inputs from the mPFC (Vertes et al., 2007). Moreover, $\sim 8 \%$ of its neurons have collaterals projecting to both CA1 and mPFC (Hoover and Vertes, 2012; Varela et al., 2014). This feature could be the neuronal correlate to the involvement of this nucleus in the synchronization and coordination of the activity of these two brain regions. Indeed, Re nucleus has been associated with the effective coupling of Hip and mPFC by theta and delta oscillations (Roy et al., 2017), and manipulation of the theta rhythm in Re nucleus has been shown to induce working memory deficits (Duan et al., 2015). Re nucleus also strongly projects to the entorhinal cortex (EC; Vertes et al., 2006), and some of the projections of Re and EC are converging onto the same pyramidal cells in CA1 (Dolleman-van der Weel et al., 2017), which may be the neural basis for a facilitation of EC-Hip information flow mediated by Re activation (Xu and Südhof, 2013). Accordingly, Re stimulation induces long-term potentiation in CA1, suggesting a potential role in hippocampal plasticity (Dolleman-Van Der Weel et al., 1997). This result was confirmed by Bertram and Zhang (1999), who demonstrated that the excitatory efferent projection from Re to CA1 allows for a direct and powerful excitation of $\mathrm{CA} 1$ and that this projection can have an excitatory effect on CA1 similar, or even greater, than that of CA3. These properties put the ReRh in an ideal situation to contribute to CA1 place cell activity.

Here, we tested the effects of ReRh lesions on spatial properties and field stability of dorsal hippocampal CA1 place cells as rats explored a familiar or a novel environment. To assess the evolution of place cell firing patterns over time, we studied the activity of a set of well identified CA1 place cells across 5 successive days, as rats were repeatedly exposed to the familiar and to the novel environment following a predefined sequence. Our results provide evidence for a contribution of the ReRh nuclei in the long-term stability of the spatial representations encoded by hippocampal CA1 place cells.

\section{Materials and Methods}

All procedures complied with both European (2010/63/UE of September 22, 2010) and French (Council Directive no. 87848) institutional rules and guidelines.

\begin{abstract}
Subjects
We used 16 male Long-Evans rats (Janvier Labs), 3 months of age, and weighing $255-275 \mathrm{~g}$ at their arrival in the laboratory. Six animals were excluded from the analyses due to misplaced tetrodes or unsuitable ReRh lesions (final number of individuals per group: ReRh, 5; SHAM, 5). Animals were housed individually in quiet facilities under a $12 \mathrm{~h}$ light/ dark cycle (light on at 07:00 A.M.) and maintained in the same temperature-controlled $\left(20^{\circ} \mathrm{C}\right)$ room for the whole duration of the experiment. The animals had ad libitum access to food and water until the beginning of the training in the pellet-chasing task, then were food deprived to $85-90 \%$ of their bodyweight during the experiments. Animals were individually handled for several minutes each day over 3 consecutive days before surgical procedures (see below), then they were handled for 1 week before behavioral experiments.
\end{abstract}

\section{Apparatus and behavioral procedures}

The familiar recording arena consisted of a dark gray cylindrical wall $50 \mathrm{~cm}$ high and $80 \mathrm{~cm}$ in diameter, placed on a gray wooden floor (Fig. $1 B)$. The three novel recording arenas were two square-shaped $(50 \mathrm{~cm}$ high and $80 \mathrm{~cm}$ in diameter) walls and one triangle-shaped $(50 \mathrm{~cm}$ high and $85 \mathrm{~cm}$ in base) walls. One square-shaped arena was painted dark gray, the other one white, as was the triangle-shaped arena (Fig. 1C). Attached to one of the internal wall was a white (in dark gray arenas) or black (in white arenas) card, $50 \mathrm{~cm}$ high and covering $90^{\circ}$ of $\operatorname{arc}$ (in the familiar circular arena) or $\sim 80 \%$ of the width of one of the walls in the square- or triangle-shaped arenas, thus providing an orienting cue for spatial navigation. The arena was surrounded by ceiling-high curtains and dimly lit from above. To promote continuous locomotion and allow a complete spatial sampling of the environment, $20 \mathrm{mg}$ food pellets (5TUL formula; TestDiet) were thrown in every $20 \mathrm{~s}$ to random locations within the arena. Each recording session lasted $10 \mathrm{~min}$. Rats were returned to their home cage between two consecutive sessions (without being disconnected from the recording setup) for a few (2-3) minutes (time required for the experimenter to clean the arena and, when required, change the arena with a different one).

\section{Surgical procedures}

NMDA permanent fiber-sparing excitotoxic lesions of Re nucleus. Subjects were anesthetized with sodium pentobarbital (50 mg/kg, i.p.), then placed in a stereotaxic frame (Kopf Instruments) where the head was held in the horizontal plane. Pentobarbital was used instead of classical ketamine/xylazine mixture to avoid any interaction between ketamine, which is a NDMA antagonist, and the NMDA per se, which was used as the neurotoxic agent inducing the lesions. Lesions targeting ReRh nuclei were made using three slow microinfusions of $0.12 \mathrm{M}$ NMDA $(0.1 \mu \mathrm{l} / \mathrm{site}$, $0.1 \mu \mathrm{l} / \mathrm{min}$; catalog \#M3262, Sigma-Aldrich) per animal using an infusion needle $(0.28 \mathrm{~mm}$ in diameter) connected to a motorized infusion pump (catalog \#CMA-100, CMA Microdialysis). NMDA was dissolved in PBS, and the sham-operated controls were infused with PBS instead of NMDA at the same coordinates. Using an angle of $15^{\circ}$, the coordinates of the three infusion sites were as follows: $\mathrm{AP}=-1.5,-2.1$, and $-2.7 \mathrm{~mm}$ from bregma; $\mathrm{DV}=-7.0,-7.1$, and $-7.2 \mathrm{~mm}$ from skull; $\mathrm{ML}=1.8$, 1.8 , and $1.9 \mathrm{~mm}$ right from midline of the sagittal sinus (Paxinos and Watson, 2007). For each infusion site, the needle was slowly removed after having been left in situ for an additional 5 min to ensure diffusion of NMDA into the cerebral parenchyma.

CA1 tetrode implantation. Approximately 2 weeks after the ReRh lesion surgery (Fig. $1 A$, timeline of the experiment), each rat was implanted with an array of eight tetrodes, each consisting of four platinum-iridium wires (90\% platinum, 10\% iridium; HML-insulated, $25 \mu \mathrm{m}$ bare wire diameter; California Fine Wire) twisted together and aiming at the CA1 layer of the dorsal hippocampus on the hemispheric side that was not crossed by the infusion needle during the lesion surgery. This surgical procedure was performed under general anesthesia with a mixture of ketamine $(98 \mathrm{mg} / \mathrm{kg})$ and xylazine $(13 \mathrm{mg} / \mathrm{kg})$ administered intraperitoneally. The tips of the tetrode bundle were placed over hippocampal CA1, $3.8 \mathrm{~mm}$ posterior to and $2.5 \mathrm{~mm}$ lateral to bregma, and $1.2 \mathrm{~mm}$ below dura mater (Paxinos and Watson, 2007). The tetrode headstage was anchored to the skull using small stainless steel screws. Embedded in dental cement with the headstage, three screws allowed to further way down the tetrodes in the rat brain when screening for place cells (Kubie, 1984).

Postoperative care. Following both surgeries, all animals received an injection of antibiotic (Clamoxyl, $50 \mathrm{mg} / \mathrm{kg}$, s.c.) and were allowed 1 week to recover in a postoperative recovery room. Passed this delay, animals were transferred back to their stabulation room before the beginning of the behavioral experiments.

\section{Brain preparation and section processing}

At the end of the experiments, rats were killed by an injection of an overdose of sodium pentobarbital (200 mg/kg, i.p.), then transcardially perfused with a $4 \%$ phosphate-buffered $(0.1 \mathrm{~m})$ paraformaldehyde solution at $4^{\circ} \mathrm{C}$. Their brains were removed, postfixed in the same fixative at $4^{\circ} \mathrm{C}$ during $2 \mathrm{~h}$, and transferred to a $30 \%$ phosphate-buffered $(0.1 \mathrm{M})$ sucrose solution for $48 \mathrm{~h}$ at $4^{\circ} \mathrm{C}$ before being snap frozen using isopentane at $-40^{\circ} \mathrm{C}$. They were then stored at $-80^{\circ} \mathrm{C}$ before further processing. Using a cryostat, coronal sections $(40 \mu \mathrm{m})$ were cut within a block of tissue extending from -0.96 to $-4.44 \mathrm{~mm}$ from bregma (according to Paxinos and Watson, 2007) to allow the evaluation of both CA1 tetrode implantation and ReRh lesions. Half of the sections were collected on gelatin-coated 
A

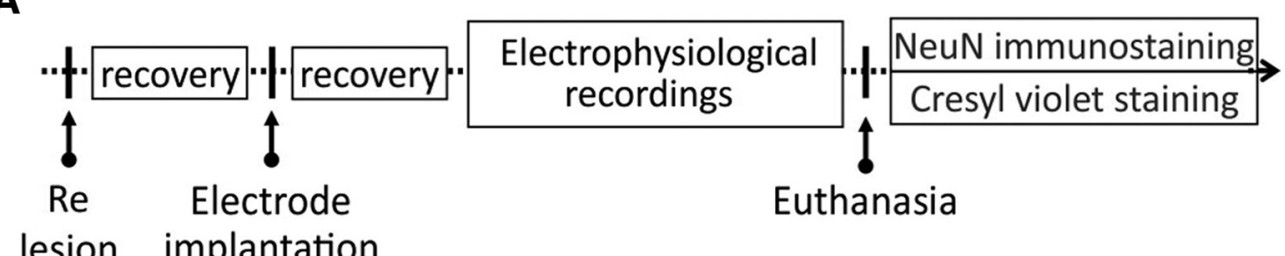

lesion implantation

in CA1

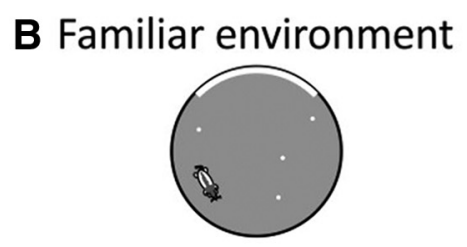

D
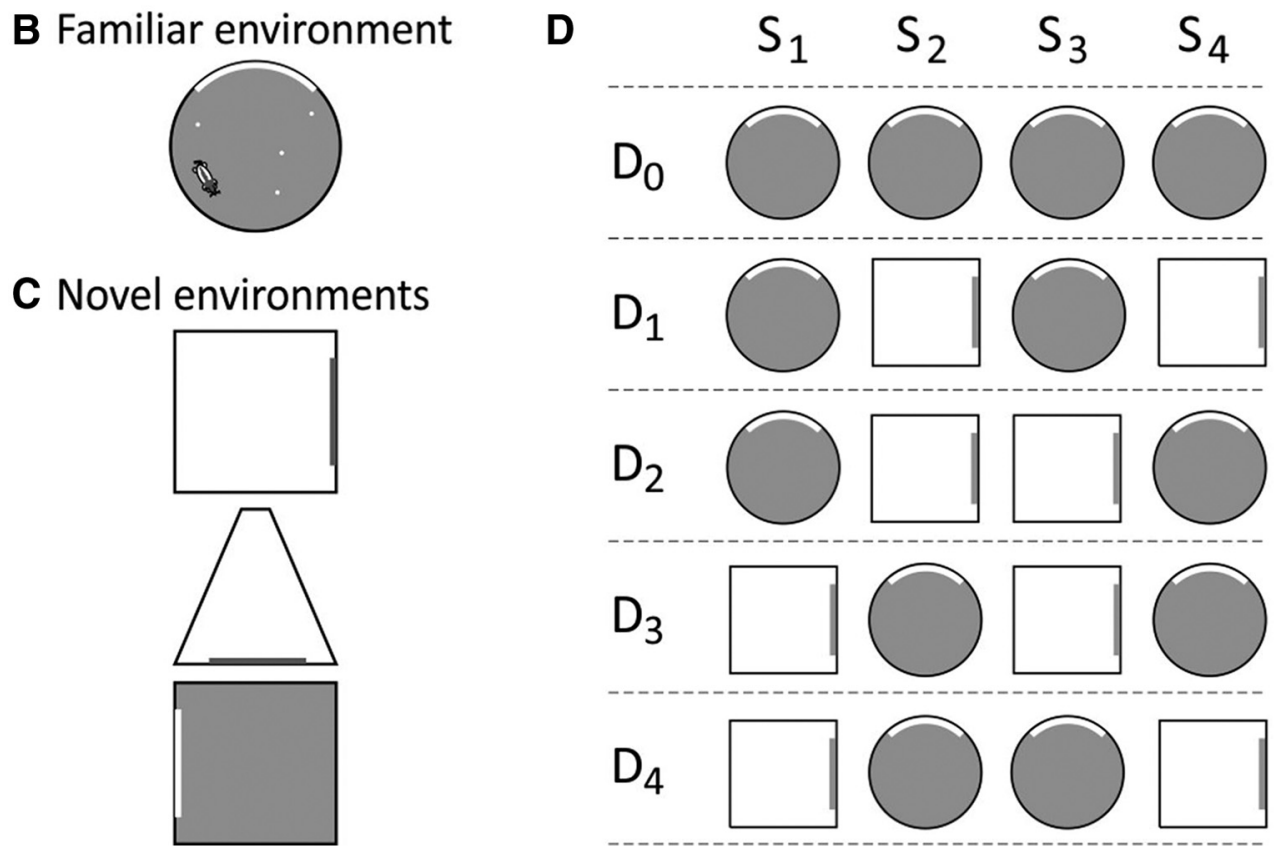

Figure 1. A, Timeline of the experiments. Following ReRh lesioning, all animals were allowed at least $10 \mathrm{~d}$ to recover. Animals were then implanted with a bundle of eight tetrodes aiming at the CA1 layer of the dorsal hippocampus. $\boldsymbol{B}$, After full recovery, animals were screened for place cells up to two times per day while they were searching for pellets scattered throughout the familiar environment. $\boldsymbol{D}$, Once several place cells were recorded simultaneously, the rats were subjected to the experimental protocol per se, which consisted of a first day of four consecutive presentations of the familiar environment (Day 0) followed by $4 \mathrm{~d}$ of pseudorandomized exposures to the familiar and a novel environment (Days $1-4$, two presentations of each environment per day). C, Up to three different novel environments were used to replicate the experiment several times for each animal.

slides and processed for cresyl violet staining (data not shown), with the other half being picked up and stored free floating in a cryoprotectant solution in 24-well plates for NeuN immunohistochemistry.

\section{NeuN immunohistochemistry protocol}

NeuN immunohistochemistry was performed using a mouse anti-NeuN antibody (1:2000; catalog \#MAB377, Millipore) as the primary antibody and a biotinylated anti-mouse horse antibody (1:500; catalog \#BA-2001, Vector Laboratories) as the secondary antibody. Briefly, sections were first rinsed three times over $10 \mathrm{~min}$ in PBS before being soaked for $1 \mathrm{~h}$ in $5 \%$ normal donkey serum (to block unspecific staining) in PBS containing $0.5 \%$ Triton $\mathrm{X}-100$. The sections were transferred into the primary anti-NeuN antibody solution for $18 \mathrm{~h}$ (overnight) at room temperature, then rinsed three times and soaked in a buffer solution containing the secondary antibody for $1 \mathrm{~h}$. After three more rinses, the staining was finally amplified and revealed using the avidin-biotin peroxidase method (Vectastain ABC Kit, Vector Laboratories) coupled to diaminobenzidine (SK-4100 kit, Vector Laboratories).

\section{Localization and extent of ReRh lesions}

Figure $2 A-D$ illustrates the location and extent of ReRh lesions in the rats that were included in analyses. Using a microscope (DMLB, Leica) equipped with a digital camera connected to a computer, NeuN slices were digitized. Then, using Free-D (a 3D reconstruction and modeling software allowing the generation, processing, and analysis of $3 \mathrm{D}$ point and surface models from stacks of 2D images), volumes of ReRh lesions and of the intact and lesioned parts of all thalamic nuclei surrounding the ventral midline thalamus were assessed. Four ReRh rats had insufficient damage to the Re area $(<50 \%)$ and/or excessive damage to the surrounding thalamic nuclei $(>15 \%)$ and were discarded from the analyses. In the five remaining ReRh rats, there was an average of $80.9 \pm 6.1 \%$ damage to the Re (range, 62.5-93.9\%). Left and right ventral Re nuclei (VRe; also sometimes called perireuniens region) were considered functionally related to the Re nucleus per se and prone to lesioning. Their condition in ReRh rats was evaluated separately, as follows: we observed $73.4 \pm 7.5 \%$ to the left VRe (range, 47.6-85.9\%) and $77.1 \pm 5.9 \%$ to the right VRe (range, 63.1-88.1\%). Based on their neuroanatomical position, their connectivity, and their role in cognitive functions, ReRh nuclei are frequently considered to form a functional group (Cassel et al., 2013). Furthermore, the location of Rh (lying just above the Re) and the lesion procedure used in our experiments make it very unlikely to ensure efficient damage to the Re without damaging the Rh. Here, we noticed $60.1 \pm 5.9 \%$ damage to the $\mathrm{Rh}$ (range, 43.2-77.4\%) in our five ReRh rats. Damage to thalamic structures other than Re, VRe, and Rh nuclei were generally minimal to modest, even when considering needle tract damage to dorsal thalamic nuclei. The average damage was $<15 \%$ for all of the nuclei surrounding the region of interest (e.g., centromedial nucleus, interanteromedial thalamic nucleus, mediodorsal nucleus, posterior hypothalamic area), except for the submedius nuclei. Lying just above the VRe in both hemispheres, laterally to the ReRh nuclei, they are particularly prone to unwanted damage in our experiments. Indeed, the mean damage was $23.7 \pm 1.0 \%$ (range, 20.3-28.6\%) in the left submedius nucleus and $19.6 \pm 1.4 \%$ (range, 14.2-24.7\%) in the right submedius 

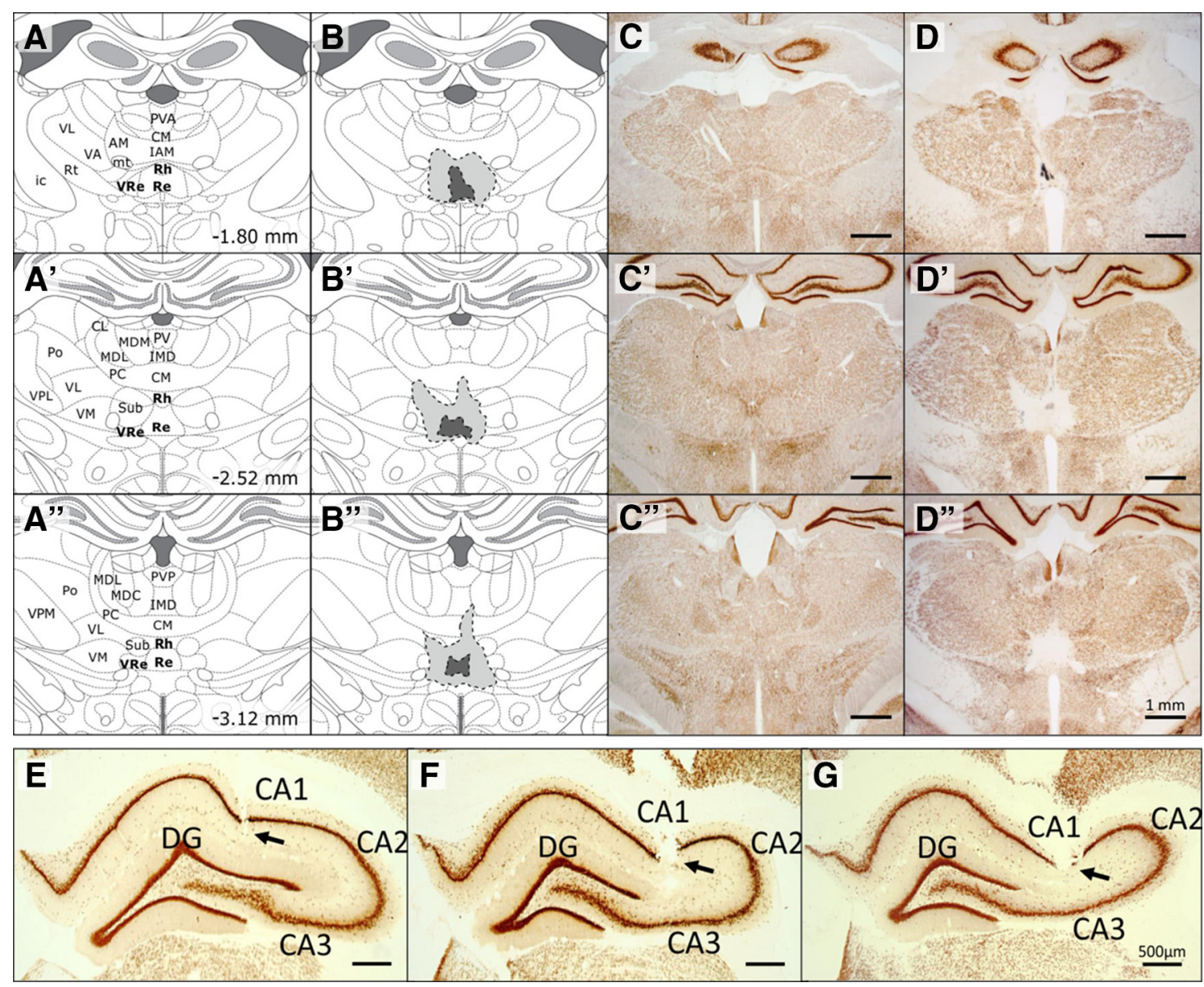

Figure 2. $A-D^{\prime \prime}$, Evaluation of the location and extent of ReRh lesions. $\boldsymbol{A}-\boldsymbol{A}^{\prime \prime}$, schematic representation of three coronal sections encompassing the rostral, median, and caudal parts of ReRh nuclei. Coordinates are given in millimeters from bregma, according to Paxinos and Watson, 2007. CL, Centrolateral thalamic nucleus; CM, central medial thalamic nucleus; IAM, interanteromedial thalamic nucleus; ic, internal capsule; IMD, intermediodorsal thalamic nucleus; MDC, central mediodorsal thalamic nucleus; MDL, lateral mediodorsal thalamic nucleus; MDM, medial mediodorsal thalamic nucleus; mt, mammillothalamic tract; $\mathrm{PC}$, paracentral thalamic nucleus; $\mathrm{Po}$, posterior thalamic nuclear group; PV, paraventricular thalamic nucleus; PVA, anterior paraventricular thalamic nucleus; PVP, posterior paraventricular thalamic nucleus; Rt, reticular thalamic nucleus; Sub, submedius thalamic nucleus; VA, ventral anterior thalamic nucleus; $V L$, ventrolateral thalamic nucleus; VM, ventromedial thalamic nucleus; VPL, ventral posterolateral thalamic nucleus. $\boldsymbol{B}-\boldsymbol{B}^{\prime \prime}$, on the same sections as in $\boldsymbol{A}-\boldsymbol{A}^{\prime \prime}$, schematic representation of the smallest (dark gray) and largest (light gray) lesions of the ventral midline thalamic nuclei (Re and Rh). $\mathbf{C}-\boldsymbol{C}^{\prime \prime}$, Typical examples of NeuN-immunostained brain sections of a SHAM animal. $\mathbf{D}-\mathbf{D}^{\prime \prime}$, Typical examples of NeuN-immunostained brain sections of ReRh animals. The three presented sections were selected to illustrate the smallest $(\boldsymbol{D})$, the largest $\left(\boldsymbol{D}^{\prime}\right)$, and an average $\left(\boldsymbol{D}^{\prime \prime}\right)$ extents of the ReRh lesions. $E-G$, Electrode locations in dorsal hippocampal area CA1 in three representative animals. Recording sites were identified by following the electrode tracks (arrowheads) through serial NeuN-immunostained sections.

nucleus. Overall, our lesions were highly specific to the ventral midline thalamic nuclei region, with minimal damage to other adjacent thalamic nuclei. Moreover, incidental unilateral damage due to the passage of the cannula to overlying areas comprising the thalamus, hippocampus, and cortex remained very limited.

\section{Histological verifications of recording sites}

Recording sites were identified by following the tetrode tracks through both cresyl violet-stained sections and NeuN-stained sections. The lowest visible tissue damage indicates the tips of the wires (Fig. $2 E-G$ ). The position of the wires at a given time (i.e., for a given electrophysiological recording) can be estimated by virtually going backward in the positioning process, consisting of lowering the tetrode during the screening phase until reaching CA1 (see Recording methods). In the animals kept for analyses, the tetrodes were located in dorsal CA1 between -3.2 and -4.1 $\mathrm{mm}$ from bregma in the rostrocaudal axis and between 2.3 and $3.1 \mathrm{~mm}$ in the mediolateral axis.

\section{Recording methods}

Beginning 1 week after surgery, the activity from each tetrode was screened daily while the rats underwent pellet-chasing sessions. If no waveform of sufficient amplitude was found, the tetrodes were lowered 25-50 $\mu \mathrm{m}$. The animals were screened one or two times per day, and a period of at least $4 \mathrm{~h}$ was left between two successive screening sessions conducted in the same rat, to guarantee the stabilization of the position of the tetrodes in the parenchyma. Once several units were recorded at the same time (i.e., at least three well isolated place cells), the recording protocol consisting of $5 \mathrm{~d}$ of alternated presentations of familiar and novel environments was initiated on the next day. Briefly, the animals were allowed to explore the familiar arena (a dark gray cylinder, Fig. $1 B$ ) or one of the three possible novel environments to which they were exposed in a fixed order (Fig. 1C, first, the white square-shaped; second, the white triangle-shaped; third, the dark gray square-shaped arenas) for four sessions per day (Fig. 1D). At least 2 weeks elapsed between the exposures to two different novel environments.

On day 0 (baseline day) of the $5 \mathrm{~d}$ recording protocol, only the familiar environment was presented four times in a row. On Days 1-4, the familiar and novel environments were presented in a predefined sequence that varied from day to day so that each environment was presented two times each day in an unpredictable order (Fig. 1D). All sessions lasted $10 \mathrm{~min}$. During this $5 \mathrm{~d}$ recording protocol, the rats were only connected once a day (they were never disconnected between the daily sessions). Screening and recording were performed with a cable attached to a powered slip ring commutator that allowed the rat to turn freely in the arena. The end of the cable (connected to the rat headstage) was equipped with opera- 
tional amplifiers and two light-emitting diodes (LEDs) for tracking the head position and direction of the rat. The signals were then carried to an adjacent room where the signals from each wire were further amplified $5000 \times$, bandpass filtered between 0.3 and $6 \mathrm{kHz}$, digitized at $32 \mathrm{kHz}$, and acquired using DataWave SciWorks software. The two LEDs were independently tracked with an overhead camera connected to a digital spot follower. Each LED was detected in a grid of pixels, allowing a resolution of $2.5 \mathrm{~cm}$ concerning head position.

Data analyses

Waveforms from the cells recorded on the same tetrode were discriminated using an Offline Sorter software (Plexon), which allows waveforms separation based on at least eight features (including spike amplitude, spike duration, and maximum and minimum spike voltage). Cluster boundaries were then refined, and outlier waveforms were removed. Interspike interval histograms were built for each isolated unit, and the whole unit was removed from analysis if it revealed the existence of interspike intervals of $<2 \mathrm{~ms}$ (limit of the refractory period). Also, only the waveforms of sufficient amplitude $(>100 \mu \mathrm{V})$ were further analyzed. These two criteria are essential for good cluster isolation. Positional firing rate maps were constructed at a pixel resolution of $2.5 \mathrm{~cm}$ according to the autoscaling scheme (Muller et al., 1987). The definition of the spatial coding of a given cell was measured using the spatial information content (Skaggs et al., 1993) and spatial coherence (Muller and Kubie, 1987). The aforementioned accurate unit isolation was confirmed by direct inspection of the positional firing rate maps. Finally, similar to previous studies (Fenton et al., 2010; Hok et al., 2012, 2013), place cells were selected for analysis if their spatial firing patterns were both location specific (spatial coherence of $\geq 0.25$ and spatial information content of $\geq 0.5 \mathrm{bits} / \mathrm{spike}$ ) and robust (average firing rate, $\geq 0.25 \mathrm{~Hz}$ ).

Overdispersion is a measure of the place cell firing variability. It was measured as described by Fenton and Muller (1998) and was similar to previous studies (Fenton et al., 2010; Hok et al., 2012, 2013). Briefly, passes through the firing field are considered as the time series of positions starting when the rat enters the field and ending when the rat leaves it. The passes needed to fulfill two conditions to be considered for analysis, as follows: each pass had to last $\geq 1 \mathrm{~s}$, and the animal trajectory must have passed through the center of the field. The observed number of spikes that occurred during a given pass was compared with a predicted number of spikes calculated on the basis of a constant distribution among the different passes. Thus, the number of spikes predicted by the session-averaged positional firing rate distribution during a pass depends only on the specific pixels visited and the time spent in those pixels, without regard to the sequential order of positions. Finally, overdispersion was measured as the variance of the distribution of $z$ values (correlation scores) computed for a set of passes (i.e., all the passes of a given recording session that reached the aforementioned criteria).

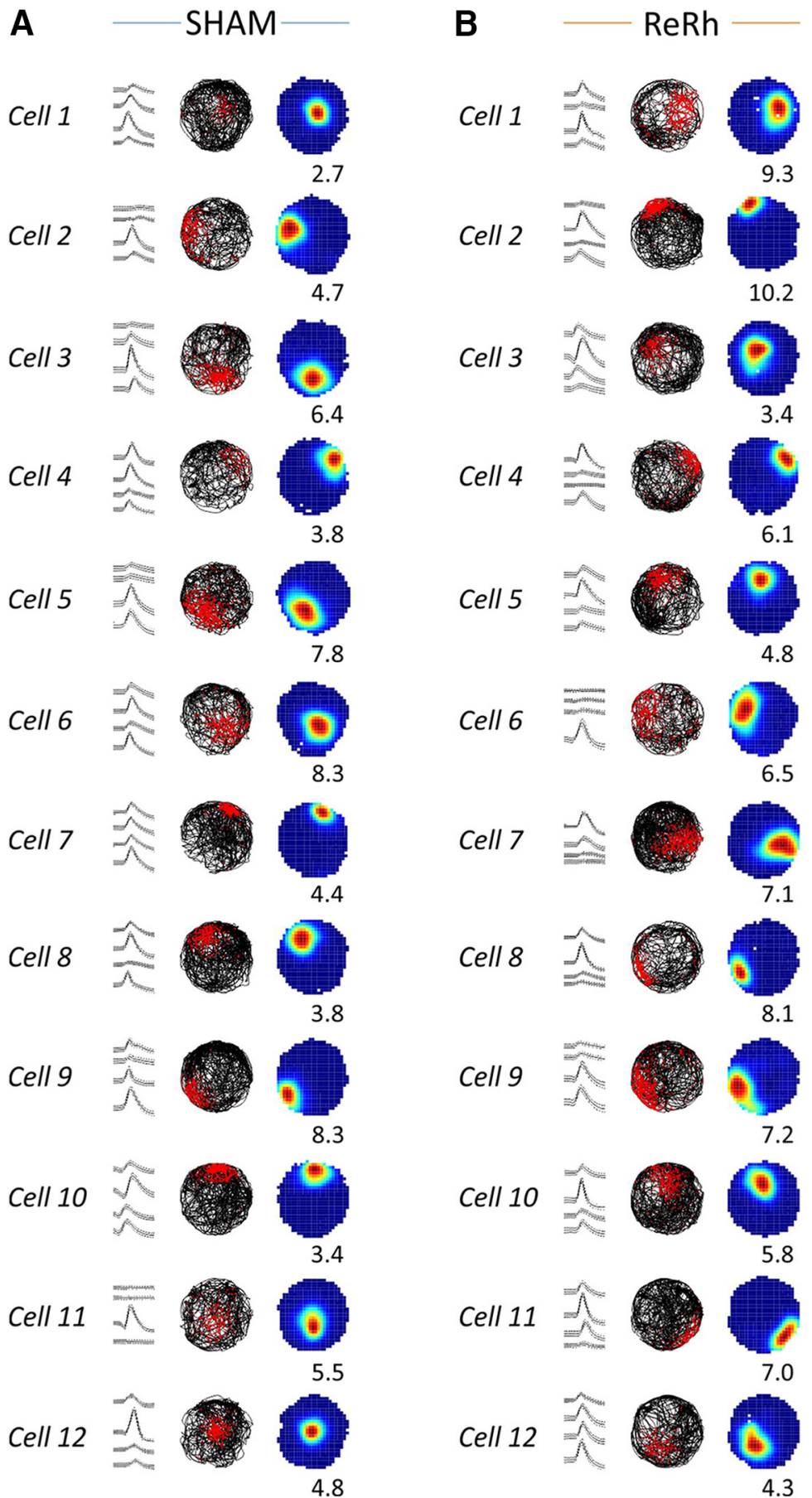

Figure 3. $\quad \boldsymbol{A}, \boldsymbol{B}$, Typical examples of place fields recorded in $\operatorname{SHAM}(\boldsymbol{A})$ and $\operatorname{ReRh}(\boldsymbol{B})$ animals. Place fields were chosen based on their spatial coherence (from best to poor through average). Left column, Mean \pm SD spike waveforms on each of the four tetrode channels. Middle column, Maps representing the trajectory of the rat (black line) with superimposed spike locations (red). Right column, Color-coded rate maps. Dark red indicates highest rate; intermediate rates are shown as orange, yellow, green, and cyan pixels from high to low; and dark blue indicates the absence of activity. The indicated rate is the grand rate within the field in hertz. One can note the absence of any clear difference in spatial selectivity between the place cells recorded in SHAM animals and in ReRh animals, as shown by the data presented in Table 1.

Experimental design and statistical analysis

The global design of the experiments is presented in Figure 1A. Familiar and novel environments as well as the pattern of exposure to the environments are shown in Figure $1 B-D$. Data were obtained from five SHAM rats and five ReRh-lesioned rats. Simple between-groups differences in behavior and electrophysiological properties of the place cell were assessed using independent $t$ tests. To compare distributions (e.g., localization of place field centroids or proportion of unstable place 
fields), we used $\chi^{2}$ tests of independence. To assess field stability or remapping between two sessions, we used a similarity score defined as the pixel-by-pixel Pearson's spatial correlation score $(r)$ between the corresponding firing rate distributions (statistical analyses were conducted on the Fisher's $z$-transforms of $r$ values). Two-way repeated-measures ANOVAs with lesion as the between-group measure and sessions as the repeated measure were used to compare across-session changes in similarity scores. When appropriate, these analyses were complemented by post hoc multiple comparisons using Newman-Keuls tests. Note that these analyses were first conducted separately on the three datasets obtained in the three different novel arenas. An overall ANOVA comparing the three datasets revealed a group effect $\left(F_{(1,95)}=11.12, p=0.001\right)$ but no arena effect $\left(F_{(2,95)}=0.214, p=0.807\right)$ or group $\times$ arena interaction $\left(F_{(2,95)}=0.16, p=0.848\right)$. Since there was no effect of the type of novel environment being used, the three datasets were pooled for further analyses. Finally, variability of place cell firing (overdispersion) was analyzed in two ways. In a first analysis, we considered all the passes of all place cells as individual data and assessed the effect of ReRh lesion using Levene's test for equality of variances. In a second analysis, we computed the mean overdispersion for each animal separately and then conducted simple independent $t$ tests to compare the ReRh and SHAM groups. Statistica software (TIBCO Software) was used to carry out all of these analyses.

\section{Results}

\section{General observations}

Rats were first trained in the dark gray cylindrical arena only, which therefore became highly familiar with repeated exposures. The recordings in the familiar arena reported here were obtained after all rats underwent at least 13 sessions (over at least $10 \mathrm{~d}$ ) in that environment. In addition, the very first exposure to a novel environment occurred after a similar number of exposures to the familiar environment in SHAM and ReRh rats $(44.8 \pm 6.9$ and $41.6 \pm 6.3$, respectively; $t_{(8)}=-0.34, p=0.742$ ).

\section{Locomotor activity is not modified following ReRh lesion}

We first determined whether ReRh lesions had any effect on locomotor activity in either the familiar or the novel environment during pellet-chasing sessions. The data are shown in Table 1. No effect of the lesion was found in the familiar environment, whether only the first exposure of the $5 \mathrm{~d}$ experimental protocol was considered (Day 0, Session $1 ; t_{(26)}=-0.38, p=0.705$ ), or all the other sessions combined $\left(t_{(306)}=-1.05, p=0.293\right)$. Similarly, no effect was found in the novel environment, whether only the first exposure was considered (in case a transient locomotor "novelty effect" would occur; Day 1 , Session $2 ; t_{(26)}=-0.68, p=$ $0.499)$, or regarding all other sessions in the novel environment $\left(t_{(194)}=0.81, p=0.418\right)$. These results are in agreement with those of previous studies showing no locomotor deficit in behavioral tasks after ReRh lesions in rats (Dolleman-van der Weel et al., 2009; Loureiro et al., 2012; Prasad et al., 2013).

\section{Characteristics of cell samples used for analyses}

We analyzed only pyramidal (complex-spike) cells with clear spatially selective firing patterns and stable activity within a recording session (Fig. 3 ). In the familiar environment, $n_{(\mathrm{SHAM})}=$ 268 and $n_{(\mathrm{ReRh})}=211$ place cells recorded during the screening phase or experimental protocol per se were kept for further analysis of the basic electrophysiological and spatial characteristics of our cell samples (Table 1). For the basic properties of place cells in the novel environment, we used $n_{\text {(SHAM) }}=78$ and $n_{(\mathrm{ReRh})}=73$ place cells that were recorded during the first exposure (Day 1, Session 2; Table 1). The main purpose of our experiment was to measure firing changes in the same cells across exposures to different environments. Accordingly, cells that were lost before the
Table 1. Behavioral (locomotor) activity and electrophysiological characteristics of CA1 place cells in SHAM and ReRh rats

\begin{tabular}{|c|c|c|c|c|}
\hline \multirow[b]{2}{*}{ Environment } & \multicolumn{2}{|l|}{ Familiar } & \multicolumn{2}{|l|}{ Novel } \\
\hline & SHAM animals & ReRh animals & SHAM animals & ReRh animals \\
\hline \multicolumn{5}{|l|}{ Locomotor activity } \\
\hline \multicolumn{5}{|l|}{ Distance travelled in } \\
\hline \multicolumn{5}{|l|}{$10 \min (m)$} \\
\hline First session & $101.37 \pm 5.88$ & $104.31 \pm 4.93$ & $81.94 \pm 4.92$ & $86.96 \pm 5.44$ \\
\hline All other sessions & $88.62 \pm 1.79$ & $91.28 \pm 1.79$ & $84.55 \pm 2.39$ & 83.14 \\
\hline \multicolumn{5}{|l|}{ Place cell characteristics } \\
\hline Sample size & 268 & 211 & 78 & 73 \\
\hline \multicolumn{5}{|c|}{ Waveform characteristics } \\
\hline Spike amplitude $(\mu \mathrm{V})$ & $157.96 \pm 4.21$ & 151.51 & $153.41 \pm 6.55$ & $150.03 \pm 16.53$ \\
\hline Spike height $(\mu V)$ & $109.40 \pm 3.70$ & 116.78 & $101.07 \pm 5.99$ & $114.14 \pm 16.85$ \\
\hline Spike half-width $(\mu s)$ & $300.49 \pm 4.86$ & $288.96 \pm 5.47$ & $317.29 \pm 9.54$ & $300.30 \pm 8.11$ \\
\hline \multicolumn{5}{|c|}{ Firing rate $(\mathrm{Hz})$} \\
\hline Overall & $1.79 \pm 0.09$ & $1.85 \pm 0.10$ & $1.95 \pm 0.17$ & $2.00 \pm 0.18$ \\
\hline Infield, GR & $5.89 \pm 0.15$ & $6.03 \pm 0.19$ & $6.91 \pm 0.39$ & $6.02 \pm 0.34$ \\
\hline Infield, CR & $13.94 \pm 0.46$ & $14.31 \pm 0.58$ & $13.82 \pm 1.08$ & $15.73 \pm 1.07$ \\
\hline \multicolumn{5}{|l|}{ Spatial characteristics } \\
\hline IC (bits/spike) & $1.98 \pm 0.06$ & $1.85 \pm 0.06$ & $1.93 \pm 0.09$ & $1.69 \pm 0.10$ \\
\hline Spatial coherence & $0.67 \pm 0.01$ & $0.66 \pm 0.01$ & $0.59 \pm 0.02$ & $0.51 \pm 0.02^{* *}$ \\
\hline $\begin{array}{c}\text { Stability within } \\
\text { session }\end{array}$ & $0.87 \pm 0.01$ & $0.86 \pm 0.01$ & $0.82 \pm 0.02$ & $0.78 \pm 0.02$ \\
\hline Field size (pixels) & $158.41 \pm 7.15$ & $0.58 \pm 7.74$ & $159.96 \pm 12.63$ & $164.60 \pm 14.40$ \\
\hline
\end{tabular}

Spike amplitude, peak relative to the most negative voltage reached during the afterhyperpolarization; Spike height, peak relative to resting potential; Spike half-width, width at half-maximal spike height; Spike peak-totrough, width between peak (highest amplitude value) and trough (lowest amplitude value); $(R$, center rate; $G R$, grand rate; $I C$, information content. Data are the mean \pm SD, unless otherwise indicated.

full session series starting on Day 0 and ending on Day 4 was completed, or whose waveforms changed so much between sessions that we could not be confident that they were the same cells, were discarded from further analysis. For these reasons, the final pool of place cells that were kept for data analyses over the $5 \mathrm{~d}$ of the study was further restricted to $n_{(\mathrm{SHAM})}=58$ and $n_{(\mathrm{ReRh})}=52$ cells in the familiar environment, and $n_{(\mathrm{SHAM})}=55$ and $n_{(\mathrm{ReRh})}=$ 46 in the novel environment.

\section{In a familiar environment, place cell spatial characteristics are not affected by ReRh lesion}

According to Dolleman-Van der Weel et al. (1997), Re nucleus is able to modulate the transmission in CA1 through both excitatory and inhibitory mechanisms (via a (subthreshold) depolarization of pyramidal cells and a (suprathreshold) excitation of inhibitory interneurons). Thus, a ReRh lesion may potentially impact CA1 pyramidal cell activity. We therefore analyzed the basic and spatial firing properties of 268 place cells recorded in SHAM animals (number of neurons per rat, 32-76) and 211 place cells found in ReRh animals (number of neurons per rat, 28-58) that were recorded during the very first recording session in the familiar dark gray cylindrical arena (Day 0, Session 1). Our data did not indicate any significant difference between ReRh and SHAM rats in spike amplitude, spike height, firing activity, information content, spatial coherence, field size, or stability within a session (Table 1).

\section{Place field distribution is not altered by ReRh lesion}

We then asked whether an ReRh lesion would affect the spatial distribution in the familiar arena (i.e., the dark gray circular arena). To do so, we first calculated the centroid of each place field (i.e., an estimate of peak firing location) according to the following formula: $X_{c}=\Sigma x_{i} r_{i} / \Sigma r_{i}$ and $Y_{c}=\Sigma y_{i} r_{i} / \Sigma r_{i}$ where the coordinates $X_{c}, Y_{c}$ of the centroid are the means of $x_{i}$ and $y_{i}$ (the $X$ and $Y$ positions of $i$ th pixel in the field) weighted by $r_{i}$, the firing 
rate in the $i$ th pixel. We then divided the surface of the arena in two equal-area zones, a central disc (middle of the arena), and an external ring (next to the wall), and compared the proportion of field centroids in these two regions in both SHAM and ReRh rats. In SHAM animals, we found 143 place cells (54.1\%) having their centroids in the external ring, while 123 centroids (45.9\%) were located in the central region. In ReRh rats, 117 centroids (59.3\%) were located in the external ring and $88(41.7 \%)$ in the central zone. These two distributions were not different $\left(\chi^{2}\right.$ test of independence: $\left.X_{(1, n=479)}^{2}=0.84, p=0.359\right)$.

\section{Remapping is not altered by ReRh lesions}

Remapping defines the capacity of the hippocampus to form a specific configuration of place fields (i.e., a unique map) for each environment. Thus, place fields can appear, disappear, or move to unpredictable locations every time the animal faces a new environment. This is a fundamental property of hippocampal neurons, since it allows the mapping of a virtually infinite number of different environments and the possibility to distinguish them from each other. Here, we tested the effects of ReRh lesions on the capacity of CA1 place cells to remap when the rats were exposed to a novel environment. To do so, we compared the similarity scores (see Materials and Methods) for two consecutive sessions recorded in the familiar and novel environments on Day 1 to Day 4 (i.e., Sessions 1 and 2 on Day 1, Sessions 1 and 2 on Day 2, Sessions 2 and 3 on Day 3, and Sessions 3 and 4 on Day 4; data not illustrated) for the $n_{\text {(SHAM) }}=58$ and $n_{(\mathrm{ReRh})}=52$ place cells recorded over the entire duration of the protocol. Mean similarity scores were $0.14 \pm 0.02$ on Day $1,0.11 \pm 0.02$ on Day $2,0.11 \pm$ 0.02 on Day 3 , and $0.13 \pm 0.02$ on Day 4 in SHAM animals; and $0.04 \pm 0.02$ on Day $1,0.09 \pm 0.01$ on Day 2, $0.09 \pm 0.01$ on Day 3 , and $0.13 \pm 0.02$ on Day 4 in ReRh rats. We found no significant alteration of remapping for the place cells recorded from ReRh rats: there was no lesion effect $\left(F_{(1,108)}=0.92, p=0.337\right)$, no day effect $\left(F_{(3,324)}=0.42, p=0.739\right)$, and no interaction between these two factors $\left(F_{(3,324)}=0.68, p=0.563\right)$. These results indicate a similar extent of place cell remapping in both SHAM and ReRh rats.

\section{Spatial coherence in a novel environment is decreased in ReRh rats}

The same analyses of basic and spatial firing properties were conducted for cells that were recorded in the novel environment for the first time (Day 1, Session $2 ; n_{\text {(SHAM) }}=78, n_{(\mathrm{ReRh})}=73$ ). Although we found no significant difference for most of the spatial parameters (i.e., information content, stability within session, field size, or firing rates; Table 1), spatial coherence was significantly decreased in ReRh rats $(0.51 \pm 0.02)$ compared with SHAM rats $\left(0.59 \pm 0.02 ; t_{(149)}=2.68, p=0.008\right)$. As spatial coherence measures the extent to which the firing rate in a pixel is predicted by the rates observed in the neighboring pixels (i.e., the local orderliness of the spatial firing pattern; Muller and Kubie, 1989), this result suggests noisier spatial firing patterns in rats exposed to a novel environment for the very first time. Note, however, that the effect of ReRh lesions on spatial coherence persisted across successive exposures to the novel environment, because an ANOVA performed on these data revealed a significant Group effect $\left(F_{(1,111)}=15.62, p=0.0001\right)$, but no Session effect $\left(F_{(7,777)}=0.914, p=0.495\right)$ or Group $\times$ Session effect $\left(F_{(7,777)}=0.607, p=0.750\right)$. None of these effects was observed for the familiar environment (Group: $F_{(1,197)}=1.36, p=0.245$;
Session: $F_{(7,1379)}=1.74, p=0.095$; Group $\times$ Session: $F_{(7,1379)}=$ $0.49, p=0.846)$.

\section{Baseline stability of place fields in a familiar environment is altered following ReRh lesions (Day 0 sessions)}

For this as well as all subsequent analyses, we focused on the 58 place cells from SHAM animals and the 52 place cells from ReRh animals that were clearly identified and active in the familiar environment during the 5 successive days of the experiment. We first assessed field stability in the familiar arena before the rats were exposed to a novel environment. On Day 0, four consecutive sessions were conducted in the familiar cylinder, thus allowing us to assess both field stability and the time course of its evolution in a well known environment (Fig. 4A). Between-session spatial correlations (Pearson's coefficient correlation, $r$ ) were used as an index of spatial similarity (see Materials and Methods). A similarity score (i.e., $r$ ) of $>0.5$ is usually considered to reflect good field stability between two sessions, whereas $r<0.5$ indicates poor stability. Using repeated-measures ANOVA, we found an overall deficit in field stability in ReRh animals compared with SHAM animals $\left(F_{(1,108)}=6.06, p=0.015\right)$. This analysis also showed a Session effect $\left(F_{(2,216)}=4.38, p=0.014\right)$, indicating an increase in field stability across successive pairs of sessions, but no Session $\times$ Group interaction $\left(F_{(2,216)}=1.44, p=0.240\right)$. Post hoc comparisons (Newman-Keuls test) revealed marginally significant differences in similarity scores between ReRh and SHAM animals for Sessions 1 and $2(p=0.057)$ as well as for Sessions 2 and $3(p=0.072)$, but no difference for Sessions 3 and 4 ( $p=$ 0.411 ; Fig. $4 B$ ). We then looked at the distributions of similarity scores (Fig. $4 C-K$ ) and investigated the possible differences between SHAM and ReRh animals in the proportion of cells seen to be unstable $(r<0.5)$. We found an increased proportion of unstable place fields in all ReRh rats between Sessions 1 and $2\left(X_{2(1, n=110)}=\right.$ $7.46, p=0.006)$ and Sessions 2 and $3\left(X_{2(1, n=110)}=5.85, p=0.016\right)$ but no difference between Sessions 3 and $4\left(X_{2(1, n=110)}=1.75, p=\right.$ $0.19)$. Together, these findings suggest an initial and overall impairment of field stability in ReRh animals, which gradually recovers with repeated exposures to the familiar arena across daily recordings, such that it eventually reaches the same level as in SHAM animals after four consecutive sessions.

\section{Field stability degrades in the familiar environment when $\mathrm{ReRh}$ rats are concurrently exposed to a novel environment over $4 \mathrm{~d}$}

We evaluated field stability in the familiar environment after the rats were also repeatedly exposed to the novel environment (Fig. $5 A$ ). We found an overall deficit of field stability (i.e., lower similarity scores) in ReRh animals compared with SHAM animals $\left(F_{(1,108)}=5.49, p=0.021\right)$, but also an effect of Day $\left(F_{(3,324)}=\right.$ $3.07, p=0.028)$ and a Day $\times$ Group interaction $\left(F_{(3,324)}=4.11\right.$, $p=0.007)$. Post hoc comparisons (Newman-Keuls test) showed that field stability in the familiar environment was high and did not vary in SHAM rats during the course of the experiment (no significant difference in any comparison). In contrast, field stability in ReRh animals was seen to decrease over days, such that it was eventually lower on Days 3 and 4 compared with Day 1 ( $p=$ 0.001 in both cases). As a result of this decrease, field stability in the familiar environment was significantly different between SHAM and ReRh animals on Day $3(p=0.048)$ and Day 4 ( $p=$ 0.035 ), but not on Days 1 and 2 (Fig. $5 B$ ). A corollary of these observations is that the proportion of cells with unstable fields $(r<0.5)$ in the familiar arena was greater in ReRh rats than in SHAM rats on Day $3\left(X_{2(1, n=110)}=9.93, p=0.002\right)$ and Day 4 
A

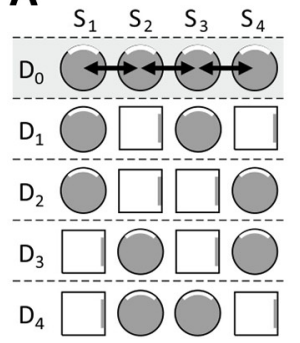

C S1 vs S2 - SHAM

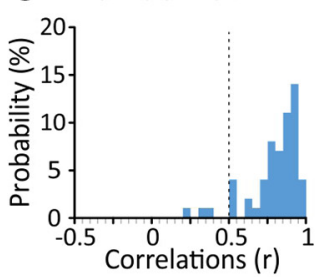

F $\quad \mathrm{S} 2$ vs $\mathrm{S} 3-\mathrm{SHAM}$

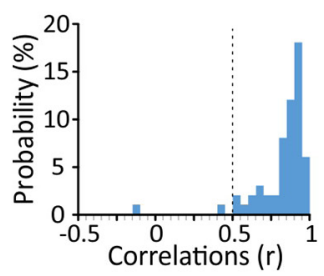

I S3 vs S4 - SHAM

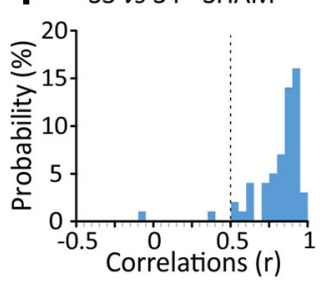

B

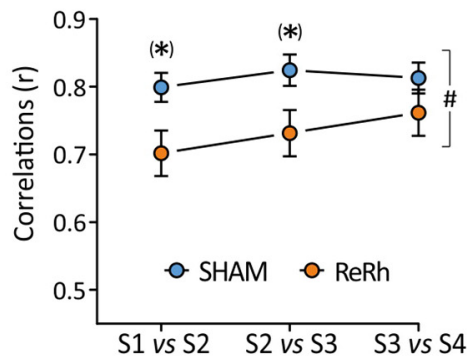

D

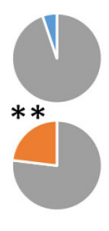

E $\quad S 1 v s \mathrm{~S} 2-\operatorname{ReRh}$

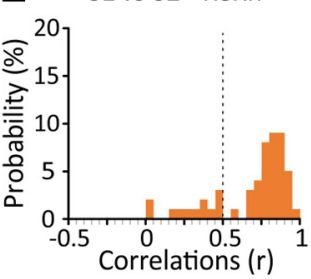

G
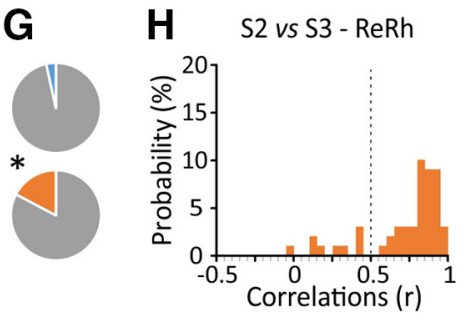

J
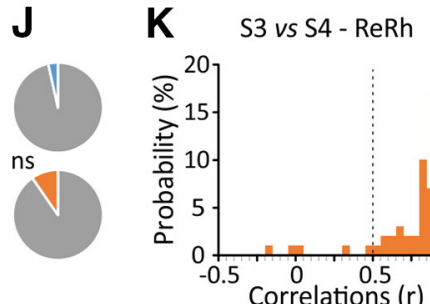

L

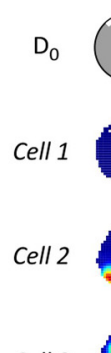

Cell 3

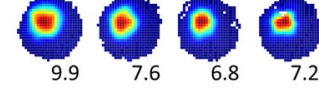

Cell 4

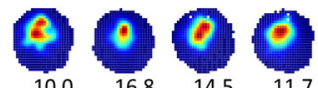

Cell 5

Cell 6

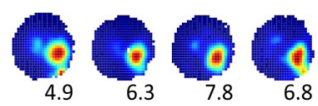

Cell 7

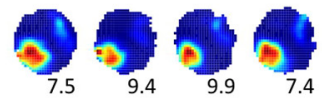

Cell 8

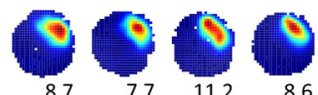

Cell 9
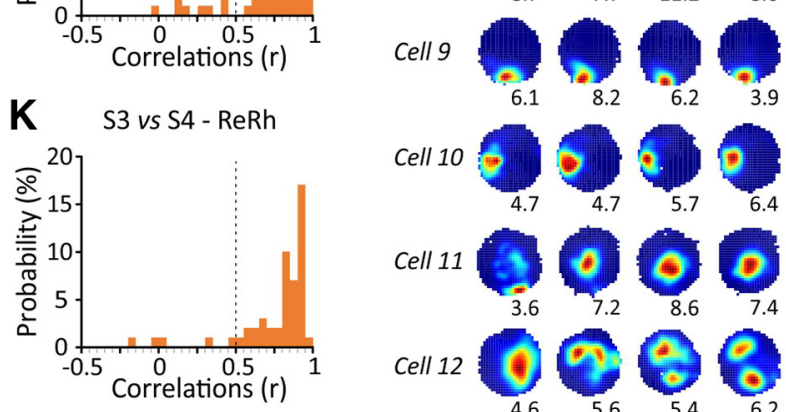

Cell 10

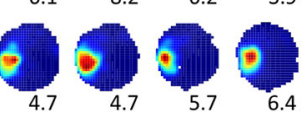

Cell 11
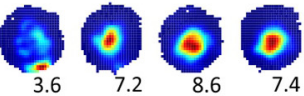

Cell 12

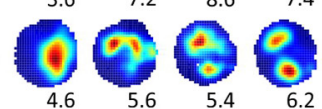

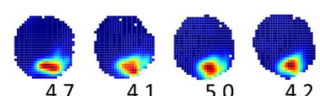

M

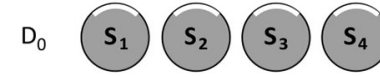

Cell 1

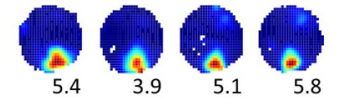

Cell 2

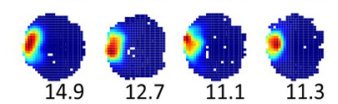

Cell 3

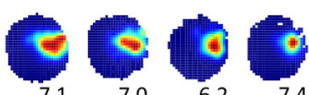

Cell 4

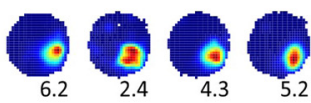

Cell 5

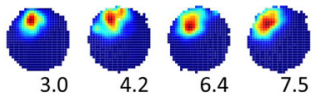

Cell 6

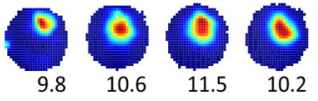

Cell 7

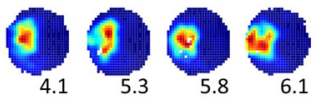

Cell 8

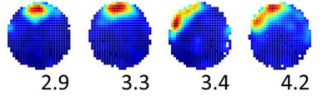

Cell 9
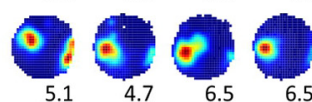

Cell 10
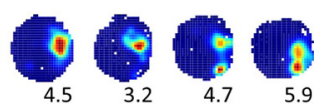

Cell 11
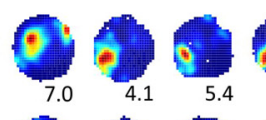

Cell 12

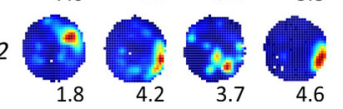

Figure 4. Effect of ReRh lesions on place field stability during Day 0 sessions (familiar environment only). $\boldsymbol{A}$, Schematic representation of the full protocol of exposure to the familiar and novel environments. The gray area indicates the sessions taken into consideration in the present figure, and arrows show the comparisons considered (i.e., $S_{1}$ vs $S_{2}, S_{2}$ vs $S_{3}$, and $S_{3}$ vs $S_{4}$ ). $\boldsymbol{B}$, Mean correlation scores ( $r$, Pearson's coefficient) indicating firing location stability between the successive $D_{0}$ sessions in SHAM and ReRh animals. \#p $=0.015$, overall group effect (ReRh vs Sham); *tendency to differ from SHAM counterparts: $p=0.057\left(S_{1}\right.$ vs $\left.S_{2}\right)$ and $p=0.072\left(S_{2} v s S_{3}\right) . C, E, F, H, I$, and $\boldsymbol{K}$, Frequency histograms depicting the distribution of the correlation scores of place cells recorded in SHAM animals $(\boldsymbol{C}, \boldsymbol{F}, \boldsymbol{I})$ and ReRh animals $(\boldsymbol{E}, \boldsymbol{H}, \boldsymbol{K})$ for the following three comparisons considered here: $\boldsymbol{S}_{1}$ vs $\boldsymbol{S}_{2}(\boldsymbol{C}, \boldsymbol{E}), \boldsymbol{S}_{2}$ vs $\boldsymbol{S}_{3}(\boldsymbol{F}, \boldsymbol{H})$, and $\boldsymbol{S}_{3}$ vs $\boldsymbol{S}_{4}(\boldsymbol{I}, \boldsymbol{K})$. A similarity score falling under a cut off of $r=0.5$, indicated by the vertical dotted line, was considered to reflect an unstable field. $\boldsymbol{D}, \boldsymbol{G}, \boldsymbol{J}$, Proportions of unstable place fields in SHAM (top) and ReRh (bottom) animals, obtained by pooling the adjacent data (based on the cutoff of $r=0.5$ ). $\boldsymbol{D}$ illustrates the data presented in $\boldsymbol{C}$ and $\boldsymbol{E} ; \boldsymbol{G}$ is based on $\boldsymbol{F}$ and $\boldsymbol{H}$; and $\boldsymbol{J}$ is based on $\boldsymbol{I}$ and $\boldsymbol{K}$. One can notice the low proportion of unstable fields in SHAM animals compared with ReRh animals and the progressive decrease of this category of cells in ReRh animals with repeated exposure to the familiar environment. ${ }^{*} p=0.016,{ }^{* *} p=0.006$ compared with SHAM counterparts. $\boldsymbol{L}, \boldsymbol{M}$, Typical examples of place cells firing activity during Day 0 sessions in SHAM $(\boldsymbol{L})$ and ReRh $(\boldsymbol{M})$ animals. These examples are classified according to their stability [i.e., from the most stable one (cell 1) to the less stable one (cell 12) for both groups] and have been selected to reflect the variability observed in the recorded population of cells.

$\left(X_{2(1, n=110)}=9.93, p=0.002\right)$, but not on Day $1\left(X_{2(1, n=110)}=\right.$ $0.17, p=0.680)$ and Day $2\left(X_{2(1, n=110)}=0.66, p=0.416\right.$; Fig. $5 C-N)$. These results reveal a progressive loss of field stability over time in ReRh animals in the familiar environment when they are concurrently exposed to another (initially novel) environment. In contrast, field similarity in SHAM animals remained consistently high over the $4 \mathrm{~d}$, indicating a robust and longlasting representation of the familiar environment (Fig. 5O,P).

Field stability is strongly impaired in the novel environment in ReRh animals

Field stability in the novel environment was assessed the same way as in the familiar environment (Fig. 6). We found an overall deficit of field stability (i.e., similarity scores) in ReRh animals compared with SHAM animals $\left(F_{(1,99)}=13.06, p=0.0005\right)$, no Day effect $\left(F_{(3,297)}=1.35, p=0.258\right)$ and no interaction of Day $\times \operatorname{Group}\left(F_{(3,297)}=0.75, p=0.522\right)$. Post hoc comparisons (Newman-Keuls test) revealed that field stability in the novel environment was high and did not vary in SHAM animals during the course of the experiment (no significant difference in any comparison, with similarity scores consistently close to 0.75 ). In contrast, similarity scores in ReRh animals were low from Day 1 (0.47), did not improve over days ( 0.60 on Day 2, 0.50 on Day 3, and 0.59 on Day 4; Fig. 6 B), and were significantly lower than in SHAM rats on Days 1 and 3 ( $p=0.005$ and $p=0.045$, respectively). However, no significant difference between SHAM and ReRh rats was seen for Days 2 and $4(p=0.118$ and $p=0.328$, respectively; Fig. $6 F-H, L-N)$. That overall decrease of field sta- 
A

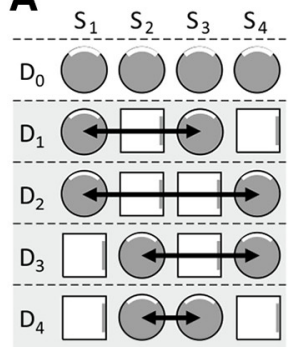

C D1: S1 vs S3 - SHAM

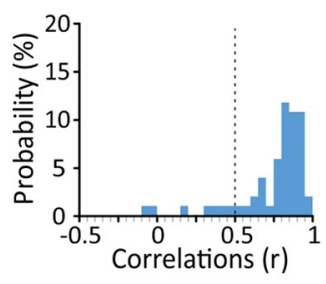

F D2: S1 vs S4 - SHAM

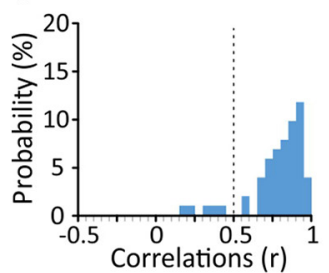

I D3: S2 vs S4 - SHAM

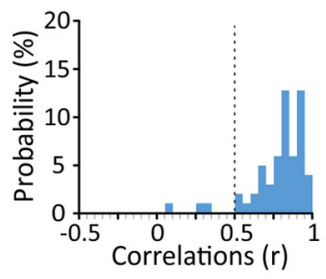

L D4: S2 vs S3 - SHAM

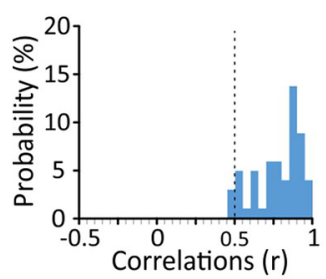

B

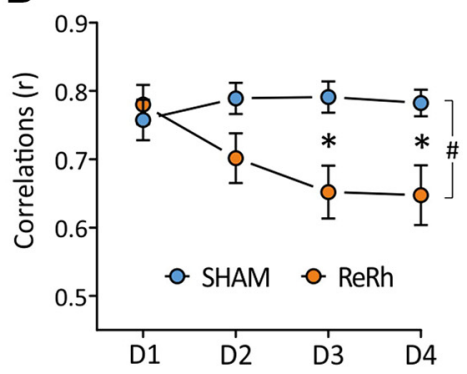

E D1: S1 vs S3-ReRh

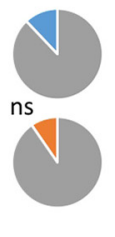

G
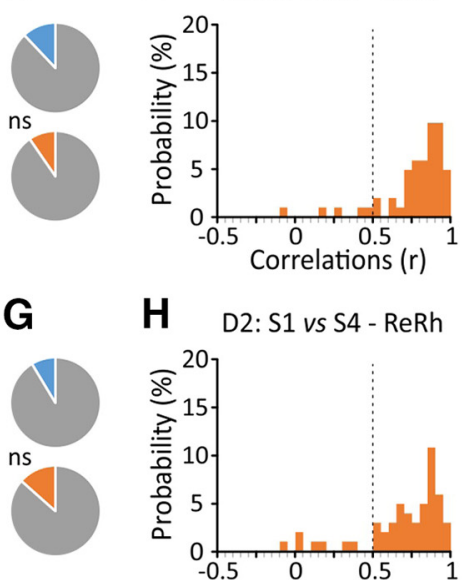

H D2: S1 vs S4 - ReRh

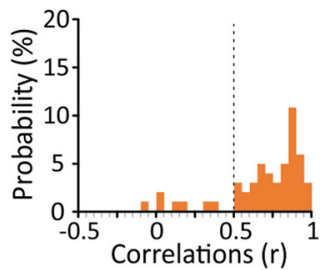

J

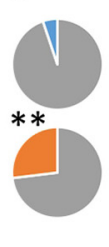

K D3: S2 vs S4 - ReRh

M N

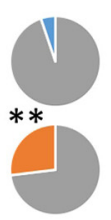

0

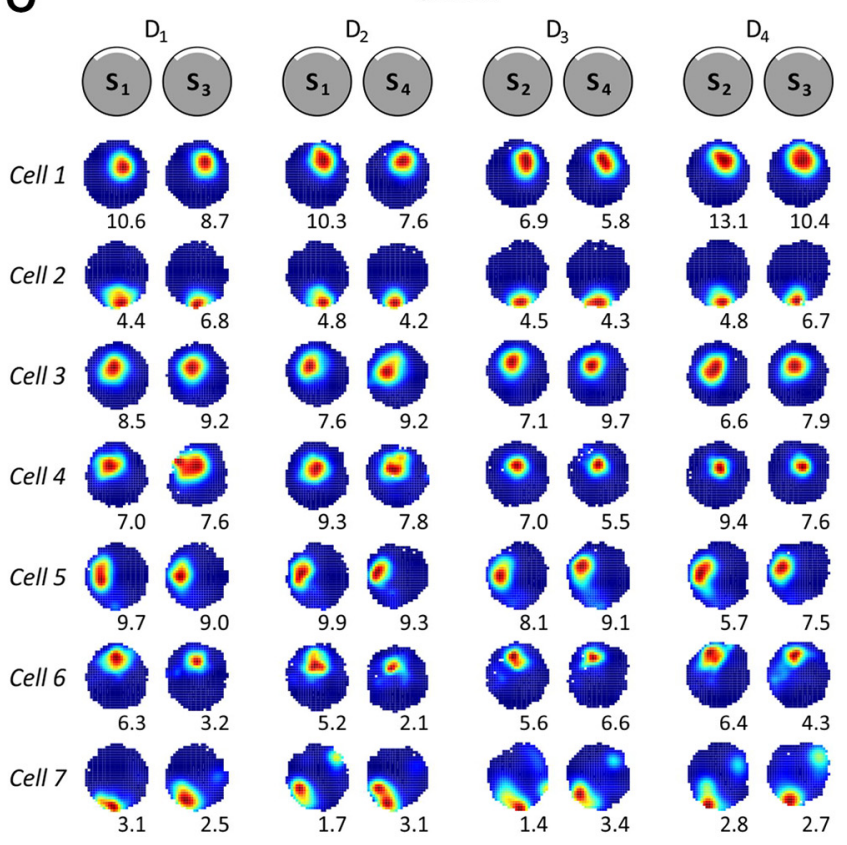

$\mathbf{P}$

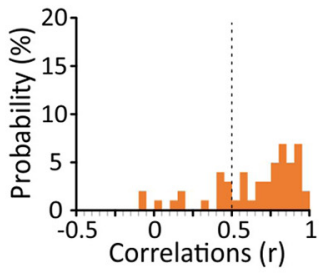

N D4: S2 vs S3-ReRh

$D_{1}$

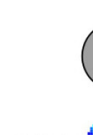

Cell 1
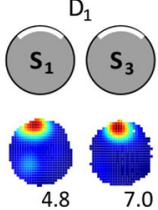

Cell 2

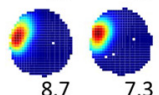

Cell 3

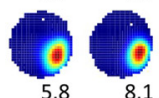

Cell 4
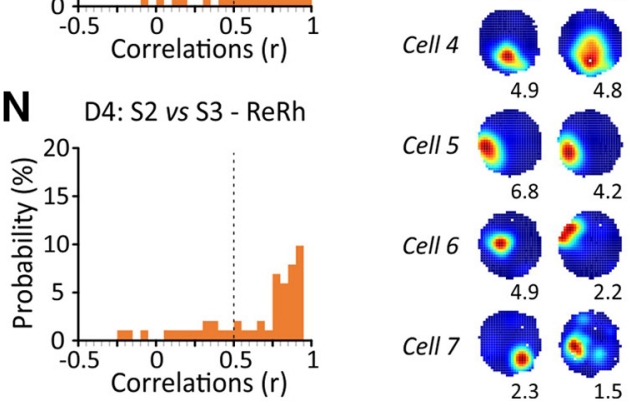

$D_{2}$

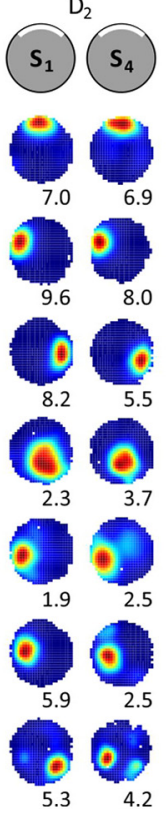

$D_{3}$
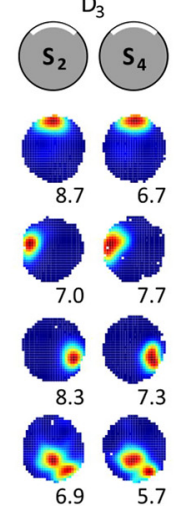

0

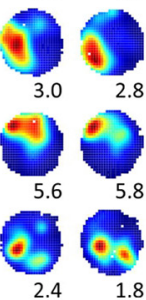

$\mathrm{D}_{4}$

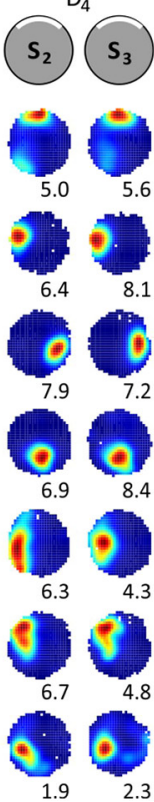

Figure 5. Effect of ReRh lesions on place field stability in the familiar environment during Days 1-4 (while the animals are also alternatively exposed to the novel environment). $A$, Schematic representation of the full experimental protocol. The gray area indicates the days taken into consideration, and arrows show the comparisons considered in the present figure. $\boldsymbol{B}$, Mean correlation $s c o r e s(r$, Pearson's coefficient) indicating firing location stability between the two sessions on a given day recorded in the familiar environment in SHAM (blue) and ReRh (orange) animals. \#p $=$ 0.021 , overall group effect (ReRh vs Sham); ${ }^{*} p=0.048$ (Day 3) and $p=0.035$ (Day 4) compared with SHAM counterparts. $C, E, F, H, I, K, L$, and $\boldsymbol{N}$, Frequency histograms depicting the distribution of the correlation scores of place cells recorded in $\operatorname{SHAM}(\boldsymbol{C}, \boldsymbol{F}, \boldsymbol{I}, \boldsymbol{L})$ and ReRh animals $(\boldsymbol{E}, \boldsymbol{H}, \boldsymbol{K}, \boldsymbol{N})$ for the $4 \mathrm{~d}$. A similarity score falling under a cutoff of $r=0.5$, indicated by the vertical dotted line, was considered to reflect an unstable field. $\boldsymbol{D}, \mathbf{G}, \boldsymbol{J}$, and $\boldsymbol{M}$, Proportions of unstable place fields in SHAM (top) and ReRh (bottom) animals obtained by pooling the adjacent data (based on the cutoff of $r=0.5$ ). $\boldsymbol{D}$ illustrates the data presented in $\boldsymbol{C}$ and $\boldsymbol{E} ; \boldsymbol{G}$ is based on $\boldsymbol{F}$ and $\boldsymbol{H} ; \boldsymbol{J}$ is based on $\boldsymbol{I}$ and $\boldsymbol{K}$; and $\boldsymbol{M}$ is based on the data presented in $\boldsymbol{L}$ and $\boldsymbol{N}$. One can notice the higher proportion of unstable fields in ReRh compared with SHAM animals at Days 3 and $4 .{ }^{* *} p=0.002$ (in both cases) compared with SHAM counterparts. $\boldsymbol{O}, \boldsymbol{P}$, Typical examples of place cells firing activity during the eight sessions recorded in the familiar environment from Day 1 to Day 4 ( 2 sessions per day) in SHAM ( $\boldsymbol{O})$ and ReRh $(\boldsymbol{P})$ animals. These examples are classified according to their stability [i.e., from the most stable one (cell 1) to the less stable one (cell 7) for both groups] and have been selected to reflect the variability observed in the recorded population of cells.

bility in ReRh rats in the novel environment was confirmed by the observation of a greater proportion of cells with unstable fields $(r<0.5)$ compared with SHAM rats on most recording days (Fig. $6 C-K$; Day $1: X_{2(1, n=101)}=15.08, p=0.0001 ;$ Day $3: X_{2(1, n=101)}=$ $7.68, p=0.006$; and Day 4: $\left.X_{2(1, n=101)}=4.94, p=0.026\right)$ but not on Day $2\left(X_{2(1, n=101)}=2.86, p=0.091\right)$. Interestingly, Day 2 was the only one to offer two successive exposures to the novel arena with no interleaved exposure to the familiar arena. Overall, these results indicate that ReRh lesions induce a severe alteration of field stability in a novel environment (Fig. 6O,P). 
A

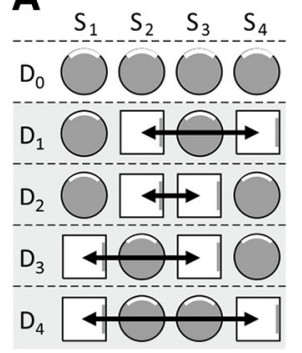

C D1: S2 vs S4 - SHAM

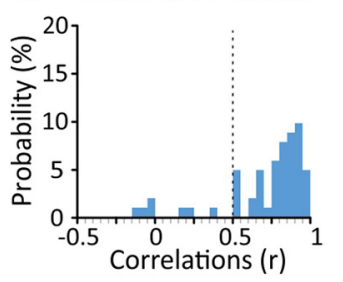

F D2: S2 vs S3 - SHAM

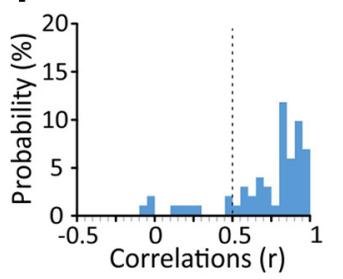

I D3: S1 vs S3 - SHAM

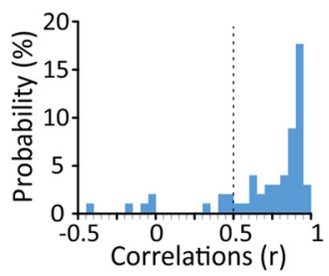

L D4: S1 vs S4 - SHAM

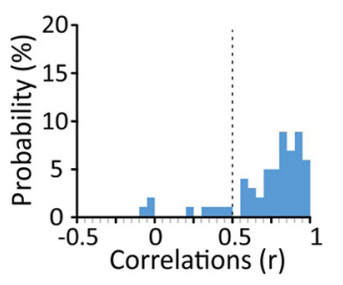

B

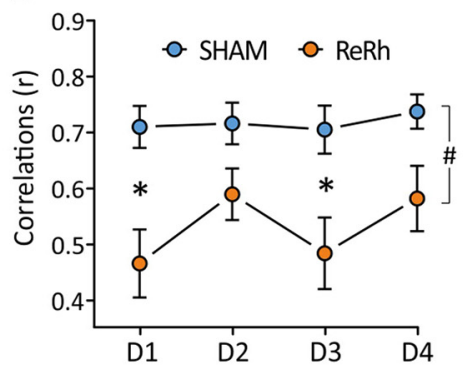

E D1: S2 vs S4 - ReRh

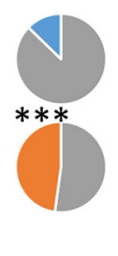

G

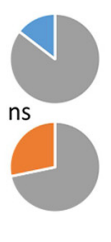

$\mathbf{J}$

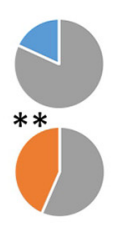

M

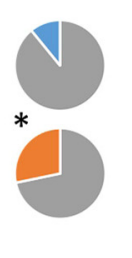

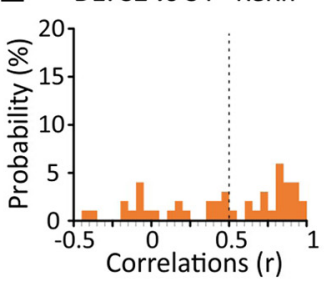

H D2: S2 vs S3 - ReRh

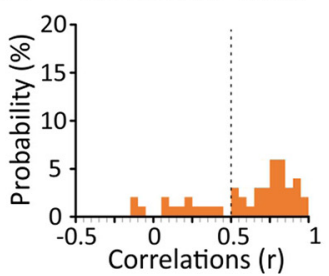

K D3: S1 vs S3 - ReRh

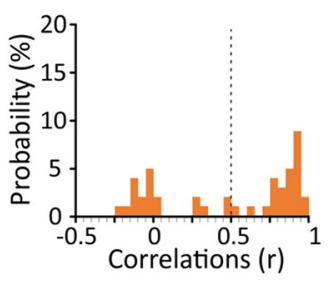

N D4: S1 vs S4 - ReRh

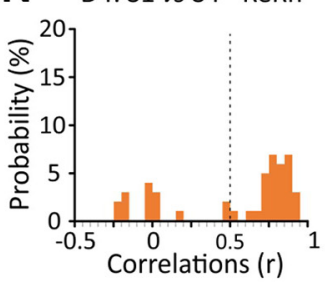

0

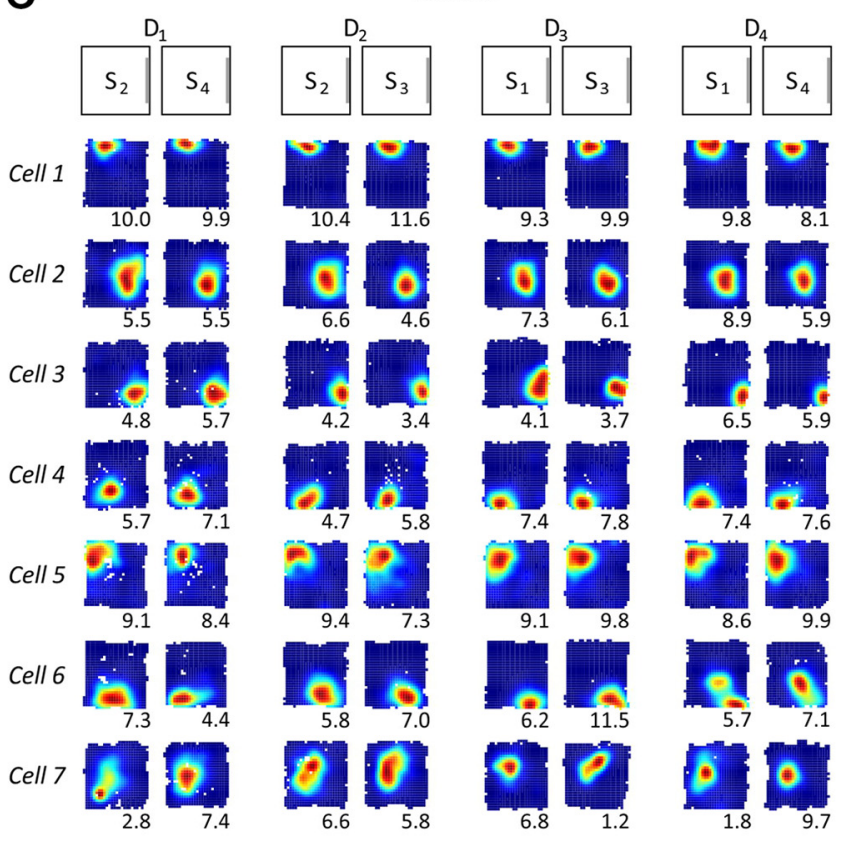

$\mathbf{P}$

ReRh
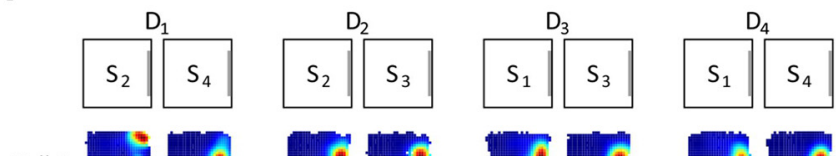

Cell 1

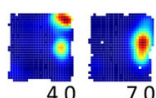

Cell 2
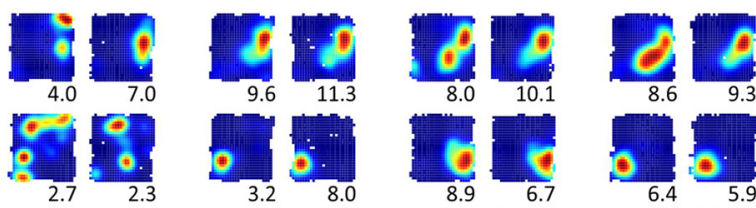

Cell 3
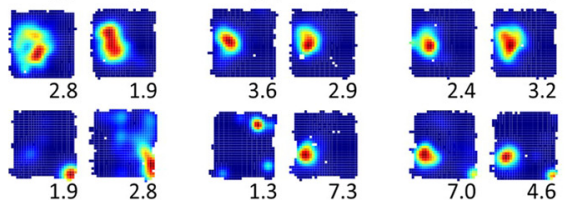

Cell 5

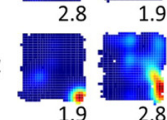

Cells

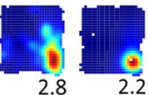

Cell 6

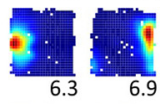

Cell 7

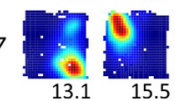

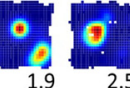
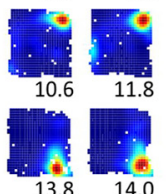
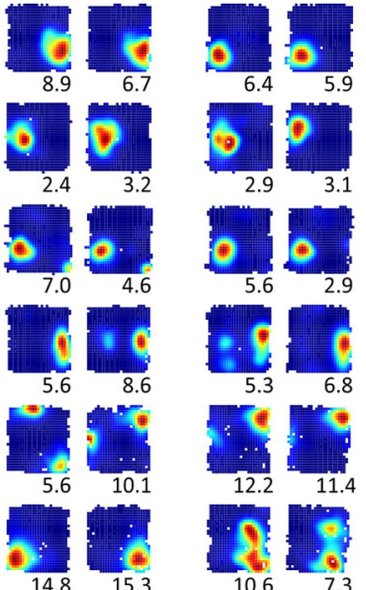

Figure 6. Effect of ReRh lesions on place field stability in the novel environment. $A$, Schematic representation of the full experimental protocol. The gray area indicates the days taken into consideration (Days 1-4), and arrows show the comparisons considered in the present figure. $\boldsymbol{B}$, Mean correlation scores ( $r$, Pearson's coefficient) indicating firing location stability between the two sessions of a given day recorded in the novel environment in SHAM (blue) and ReRh (orange) animals. $\# p=0.0005$, overall group effect (ReRh vs Sham); ${ }^{*} p=0.005$ (Day 1) and $p=0.044$ (Day 3) compared with SHAM counterparts. $C, E, F, H, I, K, L$, and $\boldsymbol{N}$, Frequency histograms depicting the distribution of the correlation scores of place cells recorded in SHAM animals $(\boldsymbol{C}, \boldsymbol{F}, \boldsymbol{I}, \boldsymbol{L})$ and ReRh animals $(\boldsymbol{E}, \boldsymbol{H}, \boldsymbol{K}, \boldsymbol{N})$ for the $4 \mathrm{~d}$. A similarity score falling under a cutoff of $r=0.5$, indicated by the vertical dotted line, was considered to reflect an unstable field. $\boldsymbol{D}, \boldsymbol{G}, \boldsymbol{J}$, and $\boldsymbol{M}$, Proportions of unstable place fields in SHAM (top) and ReRh (bottom) animals, obtained by pooling the adjacent data (based on the cutoff of $r=0.5$ ). $\boldsymbol{D}$ illustrates the data presented in $\boldsymbol{C}$ and $\boldsymbol{E} ; \boldsymbol{G}$ is based on $\boldsymbol{F}$ and $\boldsymbol{H} ; \boldsymbol{J}$ is based on $\boldsymbol{I}$ and $\boldsymbol{K}$; and $\boldsymbol{M}$ is based on the data presented in $\boldsymbol{L}$ and $\boldsymbol{N}$. One can notice the higher proportion of unstable fields in ReRh compared with SHAM animals at Days 1,3 , and 4. Statistics: ${ }^{*} p=0.026,{ }^{* *} p=0.006$, and ${ }^{* * *} p=0.0001$ compared with SHAM counterparts. $\mathbf{0}, \mathbf{P}$, Typical examples of place cells firing activity during the eight sessions recorded in the novel environment from Day 1 to Day 4 (2 sessions per day) in SHAM $(\boldsymbol{O})$ and ReRh $(\boldsymbol{P})$ animals. These examples are classified according to their stability [i.e., from the most stable one (cell 1) to the less stable one (cell 7) for both groups] and have been selected to reflect the variability observed in the recorded population of cells.

\section{Overdispersion modulation is altered following ReRh lesioning}

Overdispersion, defined as the excess variability of cell firing within the place field, is thought to reflect network-wide fluctuations and has been linked to modulation of cognitive processing in spatial tasks (Jackson and Redish, 2007; Fenton et al., 2010; Hok et al., 2012, 2013). Here, we assessed the level of overdispersion in SHAM and ReRh animals depending on the familiarity of the environment. We considered the following three major classes of recording sessions: (1) those made in the highly familiar 
environment on Day 0; (2) those performed in the familiar environment when rats were concurrently exposed to the novel environment (Days 1-4); and (3) those realized in the novel environment (Days 1-4). These results are summarized in Figure 7. Figure $7 A-C$ shows the plot of the time series of standardized firing rates for all passes through the fields of the 58 place cells from SHAM animals and 52 place cells from ReRh animals that were kept for the whole duration of the experiment. The corresponding distributions of standardized firing rates are shown in the lower part of the figures (Fig. 7D-F).

During baseline (Day 0) recordings (in the familiar environment only; Fig. $7 A, D)$, scores of overdispersion were $\sigma^{2}=3.83$ $\left[\right.$ mean $(\mu)=0.04$; number of passes $\left(n_{\text {(passes) }}\right)=3291$ for SHAM rats $]$ and $\sigma^{2}=2.38\left[\mu=0.01, n_{\text {(passes) }}=2084\right]$ for ReRh rats. Using Levene's test for equality of variances, standardized firing rates were found to be significantly less dispersed in ReRh rats than in SHAM rats (Levene test: $\mathrm{W}=105.1, p=0.00001$ ). $\mathrm{A}$ similar pattern was observed for sessions in the familiar environment with concurrent exposure to the novel environment (Days $1-4$; Fig. $7 B, E)$. Overdispersion was much reduced in ReRh rats $\left[\sigma^{2}=2.76, \mu=0.05, n_{\text {(passes) }}=3758\right]$ compared with SHAM rats $\left[\sigma^{2}=4.44, \mu=0.04, n_{\text {(passes) }}=6133\right]$, and the Levene's test again confirmed that individual scores were much less dispersed in ReRh animals than in SHAM rats $(\mathrm{W}=141.8, p=0.00001)$. In the novel environment (Fig. 7C,F), overdispersion was again significantly greater in SHAM rats $\left(\sigma^{2}=3.43, \mu=0.03, n_{\text {(passes) }}=\right.$ $4488)$ than in $\operatorname{ReRh}$ rats $\left(\sigma^{2}=2.78, \mu=-0.05, n_{\text {(passes) }}=2795\right)$, a result confirmed by the comparison of the dispersion of these data $(\mathrm{W}=23.8, p=0.00001)$. Interestingly, we noticed that overdispersion in SHAM rats was significantly reduced in the novel environment compared with the familiar environment for Days $1-4(\mathrm{~W}=47.8, p=0.00001)$, whereas overdispersion in the familiar and the novel environments were similar in ReRh animals over the same period ( $\mathrm{W}=0.004, p=0.983$ ).

To further document these findings, we analyzed the changes in overdispersion across the three types of recording sessions by calculating the overdispersion scores of each individual animal and averaging these values within each group (Fig. $7 G$ ). This analysis confirmed that overdispersion in the familiar arena was consistently weaker in ReRh rats than in SHAM rats (Day 0: $t_{(8)}=$ 2.97, $p=0.018$; Days 1-4: $\left.t_{(8)}=2.68, p=0.028\right)$ and that it was stable for the two groups (Day 0 vs Days $1-4$ : SHAM: $t_{(4)}=0.47$, $p=0.662$; ReRh: $\left.t_{(4)}=0.92, p=0.411\right)$. In contrast, there was no visible effect of the lesion in the novel environment $\left(t_{(8)}=0.86\right.$, $p=0.413$ ), due to a significant reduction of overdispersion in the novel compared with the familiar environment in SHAM rats only (Days $1-4: t_{(4)}=3.24, p=0.031$ ), while it remained stable in ReRh rats (Days 1-4: $t_{(4)}=1.42, p=0.229$ ).

To sum up, overdispersion was consistently low in ReRh animals and was not modulated by novelty. Overdispersion was greater in SHAM rats in the familiar arena, but was reduced in a novel environment.

\section{Discussion}

The present study shows that ReRh nuclei are instrumental in spatial information processing and coding of the environment. Thus, although the basic properties of place cells as well as their remapping ability were preserved following ReRh damage, we found a strong and long-lasting reduction of hippocampal place field stability. Even though such field instability was particularly pronounced in a novel environment, it was also observed in a highly familiar environment. Furthermore, in both environ- ments field instability in ReRh animals was prone to complex changes across recording sessions, suggesting that it was influenced both by time and interference effects. In addition, we found that the tendency of place cell firing to be highly variable across passes through the place field ("overdispersion") was strongly reduced in ReRh animals, and this deficit was not modulated by the novelty of the environment, as observed in SHAM animals. Together, our results indicate a substantial contribution of the ReRh nuclei to the long-term stability and specificity of distinct environmental representations at the cellular level.

\section{ReRh are required for the long-term stability of spatial representations encoded by CA1}

Evidence of a ventral midline thalamic contribution to spatial memory in rodents has already been provided in various behavioral tasks assessing spatial working memory, behavioral flexibility, goal-directed navigation, and memory consolidation (Cassel et al., 2013). In the Morris water maze, ReRh inactivations did not prevent acquisition of the task but weakened recent memory retrieval in a delayed $(24 \mathrm{~h}$ ) probe trial (Cholvin et al., 2013). When assessing a longer retention delay, ReRh lesions prevented neither task acquisition nor recent $(5 \mathrm{~d})$ memory recall, but impaired remote $(25 \mathrm{~d})$ memory performance in the probe trial (Loureiro et al., 2012; Ali et al., 2017). These results indicate a role of ReRh nuclei in the formation and/or retention of long-term, and especially remote, spatial memories. In the present study, in a well known environment presented alone (Day 0), place field stability was initially reduced in ReRh animals compared with SHAM animals but then improved across repeated exposures to finally reach control levels. This indicates a more labile (and therefore more prone to forgetting or interference) spatial representation in CA1 following ReRh lesions, but also suggests that this representation can be stabilized and reinforced with enough exposures to the learning situation. As a consequence, place field stability was similar in ReRh and SHAM rats 24 h later (Day 1; Fig. 5B). Thereby, impaired memory recall observed weeks ( $25 \mathrm{~d}$ ) after learning a spatial task in ReRh animals could be the result of less stable hippocampal representations that would remain hidden behaviorally at shorter delays such as $5 \mathrm{~d}$ (Loureiro et al., 2012; Ali et al., 2017).

\section{Ventral midline thalamic nuclei are not crucial to hippocampal remapping}

Remapping defines the capacity for the hippocampus to form a specific configuration of place fields for each environment, allowing the mapping of a virtually infinite number of different environments in distinct neural representations. Our study did not reveal any effect of ReRh damage on CA1 remapping, contrary to what is observed after medial EC manipulation. Indeed, medial EC inactivation produces substantial remapping alterations while sparing place cell spatial firing properties (Miao et al., 2015). Thus, it appears that inactivating Re or medial EC yield different outcomes on the remapping process, although the two structures project axons onto the same dendritic compartments of CA1 synapses (Dolleman-van der Weel et al., 2017). Additionally, previous work found that, following Re lesion or inactivation, trajectory-dependent CA1 place cell firing was substantially reduced in rats performing a continuous alternation task (Ito et al., 2015). The authors concluded that Re plays a crucial role in longrange communication between cortical regions important for spatial navigation and suggested that EC was involved. Xu and Südhof (2013) also proposed that the cooperative activation of Re 

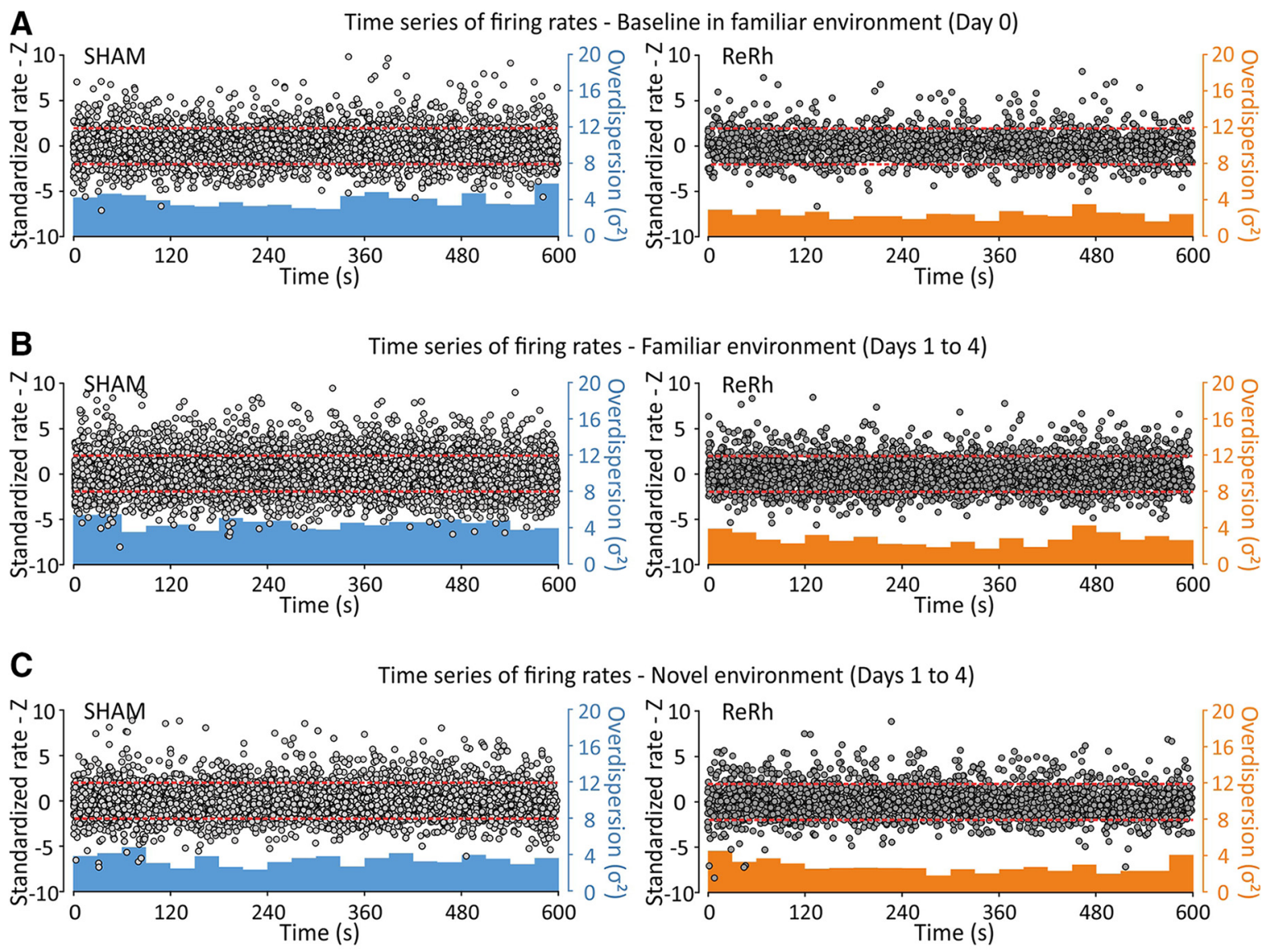

D Distribution of firing rates - Baseline (Familiar, Day 0)

E Distribution of firing rates - Familiar (Days 1 to 4)
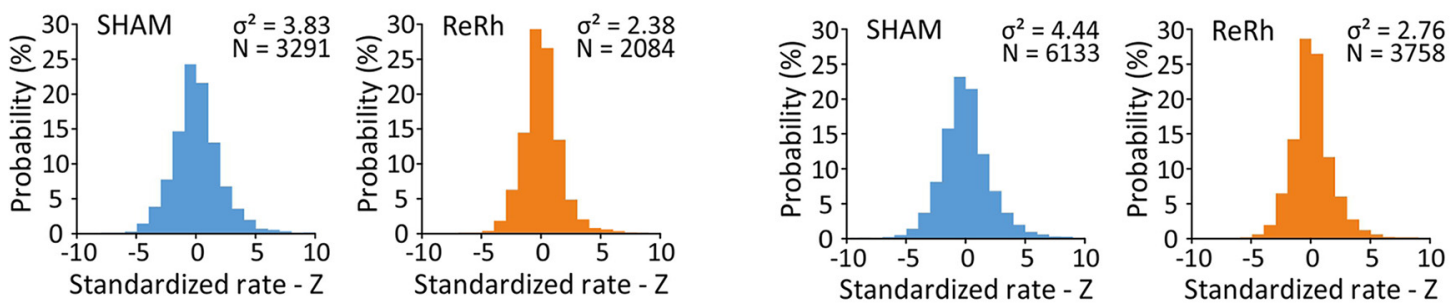

F Distribution of firing rates - Novel (Days 1 to 4 )
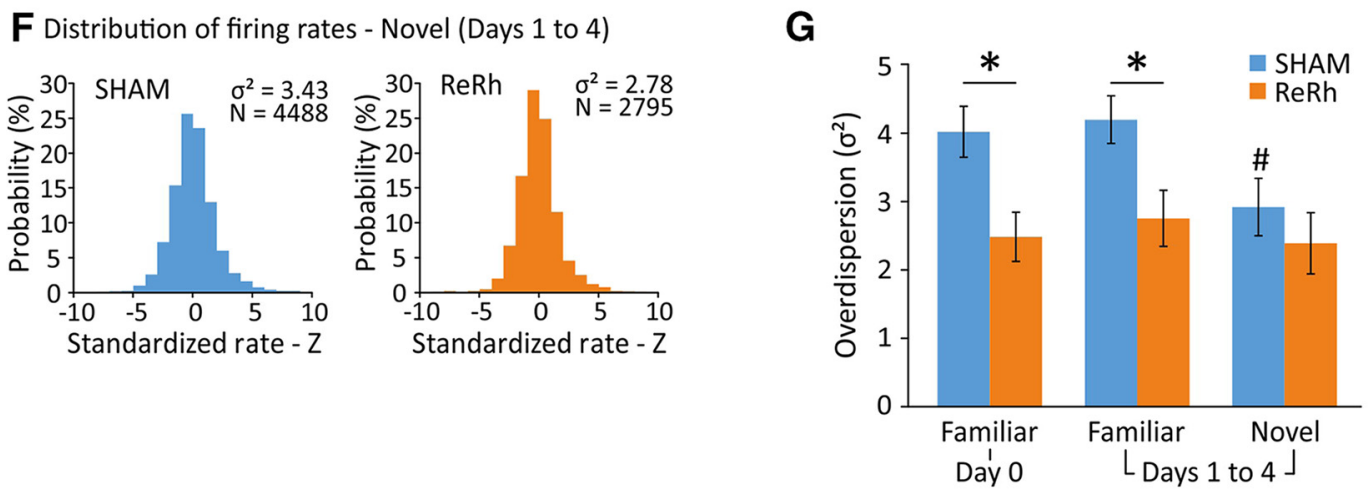

Figure 7. Dynamics of cell firing variability depending on the recording conditions: in the familiar environment only (Day 0), in the familiar environment while the rats are also alternatively exposed to the novel environment (Days 1-4), and in the novel environment (Days 1-4). A, Plot of the time series of standardized firing rates (left ordinate) for the observed firing on all passes through the center of the field of 58 (SHAM) and 52 (ReRh) place cells recorded for $10 \mathrm{~min}$. The red dotted lines indicate the 0.05 probability tails of the normal distribution ( $z=-1.96$ and $z=1.96$ ). The histograms in blue (SHAM) and orange (ReRh) show the instantaneous measure of variance (bin width $=30 \mathrm{~s}$; right ordinate). $\boldsymbol{B}, \boldsymbol{C}$, similar to $\boldsymbol{A}$, but concerning the spike firing variability observed from Day 1 to Day 4 in the familiar environment $(\boldsymbol{B})$ and in the novel environment (C). D, Histogram showing the distribution of the standardized firing rates presented in $\boldsymbol{A}$ for SHAM animals (number of passes through firing fields $=3291)$ and ReRh animals $\left(N_{\text {(passes) }}=2084\right)$. $\boldsymbol{E}, \boldsymbol{F}$, Similar to $\boldsymbol{D}$, but relative to $\boldsymbol{B}$ (familiar environment, SHAM $_{\text {(passes) }}=6133$ and ReRh (passes) $=$ 3758) and $C$ (novel environment, SHAM $_{\text {(passes) }}=4488$ and ReRh $($ passes) $=2795$ ). G, Overdispersion was also assessed by analyzing the firing variance in the set of passes through the firing fields of place cells for each animal. ${ }^{*} p=0.018$ (Day 0 ) and $p=0.028$ (Days 1-4) compared with SHAM; $\# p=0.031$ compared with the familiar environment. 
and EC inputs at the level of CA1 may reduce the threshold for synaptic plasticity, thus facilitating information transfer from EC to Hip. Through this mechanism, Re could promote the EC-Hip dialogue, and thus the incorporation of EC information in hippocampal memory representation. Although we report no remapping alteration following ReRh lesions, we did find that place cells were less stable. It is therefore possible that this outcome could be the consequence of ReRh lesion-induced reduction in the activation of $\mathrm{mEC}$ inputs to CA1, making stabilization of hippocampal representations less likely. Another possibility is that CA1 place field altered stability directly resulted from the removal of the spatially responsive cell populations present in ReRh thalamic nuclei (Jankowski et al., 2014, 2015).

\section{Ventral midline thalamic nuclei are crucial to minimize interference and discriminate among conflicting spatial representations}

Pattern separation allows similar representations to be stored in a distinct, nonoverlapping fashion. As stated by Yassa and Stark (2011), "If one were not able to perform this mnemonic discrimination, encoding new information would overwrite similar previously stored information leading to catastrophic interference." In the present study, the representation of a novel environment stabilized quickly in control rats, with place fields seen to be already stable from the second exposure onward. Conversely, in ReRh rats, stabilization of the new representation was slow and exposure to the novel environment seemingly and unexpectedly interfered with the representation of the familiar one, leading to decreased stability in both environments. The dynamics of this phenomenon suggest that interference, rather than novelty per se, was responsible for such decreased stability. Arguably, concurrent exposure to each environment was the source of interference since stability of the familiar arena representation significantly declined across days, and stability of the novel arena consistently stayed at a low level both compared with control rats and compared with the familiar arena (even though stability slightly improved after $4 \mathrm{~d}$ of exposure and reached SHAM levels). Noticeably, better field stability was observed when exposures to the novel environment were run in immediate succession (on Day 2), suggesting that novel CA1 representations in ReRh rats may stabilize in the absence of interference.

Previous work has shown that the mPFC relies on contextual information to minimize interference and to help discriminate among conflicting spatial representations (Preston and Eichenbaum, 2013). In addition, mPFC may resolve interference by modulating the relative activation of distinct memories during retrieval (Depue, 2012), as shown by impaired performance of a hippocampus-dependent spatial task and reduced separation of rule representations in the hippocampus at a cellular level following $\mathrm{mPFC}$ inactivation (Guise and Shapiro, 2017). Thus, mPFC would prevent interference by helping the hippocampus distinguish between contexts that share similar attributes. Further support for this assertion is provided by the observation that place cell stability is impaired following mPFC lesions, suggesting that mPFC normally modulates spatial responses (Kyd and Bilkey, 2003, 2005). Additionally, ReRh nuclei are reciprocally connected with mPFC and Hip, and with some reuniens neurons receiving inputs from the mPFC also directly project to CA1 (Vertes et al., 2007). Given these neuroanatomical characteristics, it is therefore possible that interrupting the information flow from the mPFC to CA1 through ReRh may have resulted in enhanced sensitivity to interferential circumstances, such as those induced by being ex- posed to different arenas in the same room with the same distal cues.

\section{The ventral midline thalamus and the regulation of attention/ awareness: a central issue to understand its implication in a wide range of cognitive functions?}

Historically, the ventral midline thalamic nuclei have long been thought to be part of a nonspecific arousing system exerting a global influence on cortical functioning (Van der Werf et al., 2002). This view has amply been challenged in the last 2 decades, leading to the proposal that ReRh is involved in a wide range of cognitive functions (Cassel et al., 2013). Surprisingly enough, during the same period, the role of these nuclei in attention has barely been investigated, even though a link between attention and place field stability has been documented in mice (Kentros et al., 2004; Muzzio et al., 2009a). This role has been suggested to rely on the nucleus reuniens as a crucial component of the frontohippocampal network (Muzzio et al., 2009b). At a behavioral level, in a five-choice reaction time task for instance, Re lesioned rats made fewer perseverative responses and fewer omissions, and collected reward quicker (Prasad et al., 2013). In a combined attention-memory task, Re rats outperformed control rats in the attention phase of the task (Prasad et al., 2017), leading the authors to suggest that visuospatial attention is enhanced following Re lesion and that Re contributes to cognitive-executive behavior through a modulation of mPFC activity. On another hand, Linley et al. (2016) recently found that ReRh lesion produced impairments on attention and behavioral flexibility using the attentional set-shifting task. Similarly, ReRh inactivation induced deficits in a strategy-shifting/spatial memory retrieval task (Cholvin et al., 2013). Overall, the data concerning the role of ventral midline thalamic nuclei in regulating attentional state during spatial tasks remain sparse.

Here, we assessed overdispersion (i.e., firing hypervariability) of CA1 place cells. Overdispersion has been shown to result from discharge fluctuations as spatial attention alternates between distal cues and local/self-motion cues (Fenton et al., 2010). The corollary to this hypothesis is that the preferential use of one class of cues should decrease overdispersion. Indeed, training rats to rely solely on distal cues to find their way to a food reward significantly reduced overdispersion (Fenton et al., 2010). Thus, overdispersion could be seen as a physiological correlate of the dynamics of attentional processes. Interestingly, exposure to a novel environment induced a reduction of overdispersion in SHAM animals, thus suggesting that novelty is associated with increased attentional focus on one class of environmental cues. Here, we showed that overdispersion was reduced in ReRh rats in both familiar and novel environments. In other words, the place cell system in ReRh rats appeared to be stuck in an attentional state associated with encoding novel information, in which one cue subset is more attended than the other. Unfortunately, the present study does not allow us to determine which cue subset was specifically attended. Hok et al. (2013) showed that mPFC inactivation decreases overdispersion in a goal-oriented navigation task using the same familiar environment as in the present study. The present results therefore suggest that ReRh lesions affect mPFC contribution to hippocampal activity, thus producing effects similar to MPFC manipulation. Overall, our findings highlight the crucial importance of ventral midline thalamic nuclei in cognitive functions requiring the interplay between $\mathrm{mPFC}$ and Hip. 


\section{References}

Ali M, Cholvin T, Muller MA, Cosquer B, Kelche C, Cassel JC, Pereira de Vasconcelos A (2017) Environmental enrichment enhances systemslevel consolidation of a spatial memory after lesions of the ventral midline thalamus. Neurobiol Learn Mem 141:108-123. CrossRef Medline

Bertram EH, Zhang DX (1999) Thalamic excitation of hippocampal CA1 neurons: a comparison with the effects of CA3 stimulation. Neuroscience 92:15-26. CrossRef Medline

Cassel JC, Pereira de Vasconcelos A, Loureiro M, Cholvin T, DalrympleAlford JC, Vertes RP (2013) The reuniens and rhomboid nuclei: neuroanatomy, electrophysiological characteristics and behavioral implications. Prog Neurobiol 111:34-52. CrossRef Medline

Cholvin T, Loureiro M, Cassel R, Cosquer B, Geiger K, De Sa Nogueira D, Raingard H, Robelin L, Kelche C, Pereira de Vasconcelos A, Cassel JC (2013) The ventral midline thalamus contributes to strategy shifting in a memory task requiring both prefrontal cortical and hippocampal functions. J Neurosci 33:8772-8783. CrossRef Medline

Depue BE (2012) A neuroanatomical model of prefrontal inhibitory modulation of memory retrieval. Neurosci Biobehav Rev 36:1382-1399. CrossRef Medline

Dolleman-Van der Weel MJ, Lopes da Silva FH, Witter MP (1997) Nucleus reuniens thalami modulates activity in hippocampal field CA1 through excitatory and inhibitory mechanisms. J Neurosci 17:5640-5650. Medline

Dolleman-van der Weel MJ, Morris RG, Witter MP (2009) Neurotoxic lesions of the thalamic reuniens or mediodorsal nucleus in rats affect non-mnemonic aspects of watermaze learning. Brain Struct Funct 213: 329-342. CrossRef Medline

Dolleman-van der Weel MJ, Lopes da Silva FH, Witter MP (2017) Interaction of nucleus reuniens and entorhinal cortex projections in hippocampal field CA1 of the rat. Brain Struct Funct 222:2421-2438. CrossRef Medline

Duan AR, Varela C, Zhang Y, Shen Y, Xiong L, Wilson MA, Lisman J (2015) Delta frequency optogenetic stimulation of the thalamic nucleus reuniens is sufficient to produce working memory deficits: relevance to schizophrenia. Biol Psychiatry 77:1098-1107. CrossRef Medline

Fenton AA, Muller RU (1998) Place cell discharge is extremely variable during individual passes of the rat through the firing field. Proc Natl Acad Sci U S A 95:3182-3187. CrossRef Medline

Fenton AA, Lytton WW, Barry JM, Lenck-Santini PP, Zinyuk LE, Kubík S, Bures J, Poucet B, Muller RU, Olypher AV (2010) Attention-like modulation of hippocampus place cell discharge. J Neurosci 30:4613-4625. CrossRef Medline

Guise KG, Shapiro ML (2017) Medial prefrontal cortex reduces memory interference by modifying hippocampal encoding. Neuron 94:183192.e8. CrossRef Medline

Hallock HL, Wang A, Griffin AL (2016) Ventral midline thalamus is critical for hippocampal-prefrontal synchrony and spatial working memory. J Neurosci 36:8372-8389. CrossRef Medline

Hembrook JR, Mair RG (2011) Lesions of reuniens and rhomboid thalamic nuclei impair radial maze win-shift performance. Hippocampus 21:815826. CrossRef Medline

Hembrook JR, Onos KD, Mair RG (2012) Inactivation of ventral midline thalamus produces selective spatial delayed conditional discrimination impairment in the rat. Hippocampus 22:853-860. CrossRef Medline

Hok V, Chah E, Reilly RB, O'Mara SM (2012) Hippocampal dynamics predict interindividual cognitive differences in rats. J Neurosci 32:35403551. CrossRef Medline

Hok V, Chah E, Save E, Poucet B (2013) Prefrontal cortex focally modulates hippocampal place cell firing patterns. J Neurosci 33:3443-3451. CrossRef Medline

Hoover WB, Vertes RP (2012) Collateral projections from nucleus reuniens of thalamus to hippocampus and medial prefrontal cortex in the rat: a single and double retrograde fluorescent labeling study. Brain Struct Funct 217:191-209. CrossRef Medline

Ito HT, Zhang SJ, Witter MP, Moser EI, Moser MB (2015) A prefrontalthalamo-hippocampal circuit for goal-directed spatial navigation. Nature 522:50-55. CrossRef Medline

Jackson J, Redish AD (2007) Network dynamics of hippocampal cellassemblies resemble multiple spatial maps within single tasks. Hippocampus 17:1209-1229. CrossRef Medline

Jankowski MM, Islam MN, Wright NF, Vann SD, Erichsen JT, Aggleton JP,
O'Mara SM (2014) Nucleus reuniens of the thalamus contains head direction cells. eLife 3:e03075. CrossRef Medline

Jankowski MM, Passecker J, Islam MN, Vann S, Erichsen JT, Aggleton JP, O'Mara SM (2015) Evidence for spatially-responsive neurons in the rostral thalamus. Front Behav Neurosci 9:256. CrossRef Medline

Kentros CG, Agnihotri NT, Streater S, Hawkins RD, Kandel ER (2004) Increased attention to spatial context increases both place field stability and spatial memory. Neuron 42:283-295. CrossRef Medline

Kubie JL (1984) A driveable bundle of microwires for collecting single-unit data from freely-moving rats. Physiol Behav 32:115-118. CrossRef Medline

Kyd RJ, Bilkey DK (2003) Prefrontal cortex lesions modify the spatial properties of hippocampal place cells. Cereb Cortex 13:444-451. CrossRef Medline

Kyd RJ, Bilkey DK (2005) Hippocampal place cells show increased sensitivity to changes in the local environment following prefrontal cortex lesions. Cereb Cortex 15:720-731. CrossRef Medline

Layfield DM, Patel M, Hallock H, Griffin AL (2015) Inactivation of the nucleus reuniens/rhomboid causes a delay-dependent impairment of spatial working memory. Neurobiol Learn Mem 125:163-167. CrossRef Medline

Linley SB, Gallo MM, Vertes RP (2016) Lesions of the ventral midline thalamus produce deficits in reversal learning and attention on an odor texture set shifting task. Brain Res 1649:110-122. CrossRef Medline

Loureiro M, Cholvin T, Lopez J, Merienne N, Latreche A, Cosquer B, Geiger K, Kelche C, Cassel JC, Pereira de Vasconcelos A (2012) The ventral midline thalamus (reuniens and rhomboid nuclei) contributes to the persistence of spatial memory in rats. J Neurosci 32:9947-9959. CrossRef Medline

McKenna JT, Vertes RP (2004) Afferent projections to nucleus reuniens of the thalamus. J Comp Neurol 480:115-142. CrossRef Medline

Miao C, Cao Q, Ito HT, Yamahachi H, Witter MP, Moser MB, Moser EI (2015) Hippocampal remapping after partial inactivation of the medial entorhinal cortex. Neuron 88:590-603. CrossRef Medline

Moser MB, Rowland DC, Moser EI (2015) Place cells, grid cells, and memory. Cold Spring Harb Perspect Biol 7:a021808. CrossRef Medline

Muller RU, Kubie JL (1987) The effects of changes in the environment on the spatial firing of hippocampal complex-spike cells. J Neurosci 7:19511968. Medline

Muller RU, Kubie JL (1989) The firing of hippocampal place cells predicts the future position of freely moving rats. J Neurosci 9:4101-4110. Medline

Muller RU, Kubie JL, Ranck JB Jr (1987) Spatial firing patterns of hippocampal complex-spike cells in a fixed environment. J Neurosci 7:19351950. Medline

Muzzio IA, Levita L, Kulkarni J, Monaco J, Kentros C, Stead M, Abbott LF, Kandel ER (2009a) Attention enhances the retrieval and stability of visuospatial and olfactory representations in the dorsal hippocampus. PLoS Biol 7:e1000140. CrossRef Medline

Muzzio IA, Kentros C, Kandel E (2009b) What is remembered? Role of attention on the encoding and retrieval of hippocampal representations. J Physiol 587:2837-2854. CrossRef Medline

O'Keefe J, Nadel L (1978) The hippocampus as a cognitive map. Oxford: Clarendon.

Paxinos G, Watson C (2007) The rat brain in stereotaxic coordinates, Ed 6 . San Diego: Academic.

Prasad JA, Macgregor EM, Chudasama Y (2013) Lesions of the thalamic reuniens cause impulsive but not compulsive responses. Brain Struct Funct 218:85-96. CrossRef Medline

Prasad JA, Abela AR, Chudasama Y (2017) Midline thalamic reuniens lesions improve executive behaviors. Neuroscience 345:77-88. CrossRef Medline

Preston AR, Eichenbaum H (2013) Interplay of hippocampus and prefrontal cortex in memory. Curr Biol 23:R764-R773. CrossRef Medline

Roy A, Svensson FP, Mazeh A, Kocsis B (2017) Prefrontal-hippocampal coupling by theta rhythm and by $2-5 \mathrm{~Hz}$ oscillation in the delta band: the role of the nucleus reuniens of the thalamus. Brain Struct Funct 222: 2819-2830. CrossRef Medline

Skaggs W, McNaughton L, Gothard K, Markus E (1993) An informationtheoretic approach to deciphering the hippocampal code. In: Advances 
in neural information processing systems 5 (NIPS 1992) (Hanson SJ, Cowan JD, Giles CL, eds), pp 1030-1037. San Mateo, CA: Morgan Kaufmann.

Van der Werf YD, Witter MP, Groenewegen HJ (2002) The intralaminar and midline nuclei of the thalamus. Anatomical and functional evidence for participation in processes of arousal and awareness. Brain Res Rev 39:107-140. CrossRef Medline

Varela C, Kumar S, Yang JY, Wilson MA (2014) Anatomical substrates for direct interactions between hippocampus, medial prefrontal cortex, and the thalamic nucleus reuniens. Brain Struct Funct 219:911-929. CrossRef Medline

Vertes RP (2002) Analysis of projections from the medial prefrontal cortex to the thalamus in the rat, with emphasis on nucleus reuniens. J Comp Neurol 442:163-187. CrossRef Medline

Vertes RP, Hoover WB, Do Valle AC, Sherman A, Rodriguez JJ (2006) Efferent projections of reuniens and rhomboid nuclei of the thalamus in the rat. J Comp Neurol 499:768-796. CrossRef Medline

Vertes RP, Hoover WB, Szigeti-Buck K, Leranth C (2007) Nucleus reuniens of the midline thalamus: link between the medial prefrontal cortex and the hippocampus. Brain Res Bull 71:601-609. CrossRef Medline

Xu W, Südhof TC (2013) A neural circuit for memory specificity and generalization. Science 339:1290-1295. CrossRef Medline

Yassa MA, Stark CE (2011) Pattern separation in the hippocampus. Trends Neurosci 34:515-525. CrossRef Medline 